\title{
On canonical triangulations of once-punctured torus bundles and two-bridge link complements
}

\author{
FRANÇOIS GUÉRITAUD \\ APPENDIX A BY DAVID FUTER
}

\begin{abstract}
We prove the hyperbolization theorem for punctured torus bundles and two-bridge link complements by decomposing them into ideal tetrahedra which are then given hyperbolic structures, following Rivin's volume maximization principle.
\end{abstract}

57M50; 57M27

À la mémoire de Pierre Philipps

\section{Introduction}

Let $T:=\left(\mathbb{R}^{2} \backslash \mathbb{Z}^{2}\right) / \mathbb{Z}^{2}$ be the once-punctured torus endowed with its differential structure and an orientation. The group $G$ of isotopy classes of orientation-preserving diffeomorphisms $\varphi: T \rightarrow T$ (or the mapping class group of $T$ ) is identified as $G \simeq$ $\mathrm{SL}_{2}(\mathbb{Z})$, so each such map $\varphi$ has well-defined eigenvalues in $\mathbb{C}$. For $[\varphi]$ in $G$, define the punctured torus bundle

$$
V_{\varphi}:=T \times[0,1] / \sim
$$

where $\sim$ identifies $(x, 0)$ with $(\varphi(x), 1)$ for all $x$ in $T$. Then $V_{\varphi}$ is a differentiable oriented 3-manifold, well-defined up to diffeomorphism. Thurston's Hyperbolization Theorem [21] implies the following theorem as a very special case.

Theorem 1.1 If $\varphi$ has two distinct real eigenvalues, the punctured torus bundle $V_{\varphi}$ admits a finite-volume, complete hyperbolic metric.

The aim of this paper is to prove Theorem 1.1 by elementary and, to some extent, constructive arguments. The strategy is to exhibit a canonical, geodesic triangulation $\mathcal{H}$ of $V_{\varphi}$ into ideal tetrahedra (hyperbolic tetrahedra whose vertices are at infinity).

Combinatorially, $\mathcal{H}$ (sometimes called the Floyd-Hatcher or monodromy triangulation) is found by expressing a certain conjugate of $\pm \varphi$ as a product of positive transvection matrices. Once such combinatorial data for a triangulation is given, the problem of 
making it hyperbolic lends itself to two approaches. One is complex, explicit and "local": cross-ratio computations, particular hyperbolic isometries, etc (see eg Neumann and Zagier [20]). The other approach, first described by Rivin [22], de Verdière [25] and Casson, is real-analytic and "global": in order to make the structure complete, one kills its monodromy by maximizing the total hyperbolic volume (but combinatorial obstructions might arise). In the case of $V_{\varphi}$, the combinatorial structure of $\mathcal{H}$ is sufficiently well-understood to allow a nice interplay between the two approaches, yielding useful "medium-range" results (Section 8). The philosophy of such results is that if the structure with maximal volume is noncomplete, it should still be complete at "most" places, enabling us to make geometric statements.

Akiyoshi [4], combining the methods of Akiyoshi, Sakuma, Wada and Yamashita [5] and Minsky [18], proved that the "combinatorially canonical" triangulation $\mathcal{H}$ must also be "geometrically canonical," ie topologically dual to the Ford-Voronoi domain of $V_{\varphi}$. Lackenby [13], assuming the existence of the hyperbolic metric, derived the same result by a normal surface argument. In [10], we apply the methods of the present paper to extend the Akiyoshi-Lackenby theorem to quasifuchsian groups (where pleating laminations of the convex core replace the attractive and repulsive laminations of the monodromy $\varphi$ ).

Knowing the space of angle structures also allows for easy volume estimates, some of which are worked out in the Appendix: these estimates make the constants of Brock [8] explicit (and sharp) for the class of manifolds under consideration. Although the main results of the present paper are known, our ambition is to demonstrate that hyperbolic geometry and combinatorics (of the curve complex, say) can interact more intimately than at the level of coarse geometry, a phenomenon which seems to extend beyond punctured torus groups and begs to be further explored. Other references closely related to this subject include the beautiful article of Akiyoshi, Sakuma, Wada and Yamashita [6], which builds on the work of Jørgensen and partly motivated our work [10], and the examples compiled in Alestalo and Helling [7], Helling [11] and Koch [12].

The converse of Theorem 1.1 is true. If the trace $\tau$ of the monodromy map $\varphi$ is in $\{-1,0,1\}$, then $[\varphi]$ has finite order and $V_{\varphi}$ is Seifert fibered. If $\tau= \pm 2$, then $\varphi$ preserves a nontrivial simple closed curve $\gamma$ (parallel to a rational eigenvector) in the punctured torus, and $\gamma$ defines an incompressible torus or Klein bottle in $V_{\varphi}$. In any case we get a topological obstruction to the existence of the hyperbolic metric.

An attempt to be self-contained will be made in proving Theorem 1.1. The proof will deal primarily with the case where the eigenvalues of $\varphi$ are positive. The other case is only a minor variant (in particular, $V_{-\varphi}$ can be obtained by ungluing the metric tetrahedra of $V_{\varphi}$ and regluing them in a slightly different way). 
Section 2 is standard and recalls the classification of conjugacy classes in $\mathrm{SL}_{2}(\mathbb{Z})$ in order to define the triangulation $\mathcal{H}$. The latter is studied in greater detail in Section 3 and Section 4. Positive angles for $\mathcal{H}$ (a "linear hyperbolic structure") are provided in Section 5. Section 6 explains the role played by hyperbolic volume maximization, allowing us to deal with the easy cases in Section 7. Section 8 presents the essential geometric lemma for the final attack, carried out in Section 9. Section 10 is devoted to a numerical example. In Section 11, we quickly recall the connection between once-punctured tori and 4-punctured spheres. In the Appendix, David Futer builds on that connection to prove an analogue of Theorem 1.1 for the complements of two-bridge links and computes geometric estimates.

The symbol "=" is preceded (resp. followed) by a colon $(:=$, resp. =:) when the equality serves as a definition for its left (resp. right) member.

I would like to thank Francis Bonahon and Frédéric Paulin for numerous insights and the great improvements this paper owes to them. Exciting discussions with David Futer and with Makoto Sakuma also gave invaluable input. This paper reached its pre-final form during a stay at the Institut Bernoulli (EPFL, Lausanne) for whose kind hospitality I express my deep gratitude. Finally, thanks are due to the referee for many helpful comments. This work was partially supported by NSF grant DMS-0103511.

\section{Conjugacy in $\mathrm{SL}_{2}(\mathbb{Z})$ and the Farey tesselation}

Proposition 2.1 Let $\varphi$ be an element of $\mathrm{SL}_{2}(\mathbb{Z})$ with two distinct eigenvalues in $\mathbb{R}_{+}^{*}$. Then the conjugacy class of $\varphi$ in $\mathrm{SL}_{2}(\mathbb{Z})$ contains an element of the form

$$
A \varphi A^{-1}=\left(\begin{array}{cc}
1 & a_{1} \\
0 & 1
\end{array}\right)\left(\begin{array}{cc}
1 & 0 \\
b_{1} & 1
\end{array}\right)\left(\begin{array}{cc}
1 & a_{2} \\
0 & 1
\end{array}\right)\left(\begin{array}{cc}
1 & 0 \\
b_{2} & 1
\end{array}\right) \cdots\left(\begin{array}{cc}
1 & a_{n} \\
0 & 1
\end{array}\right)\left(\begin{array}{cc}
1 & 0 \\
b_{n} & 1
\end{array}\right)
$$

where $n>0$ and the $a_{i}$ and $b_{i}$ are positive integers. Moreover, the right hand side is unique up to cyclic permutation of the factors $\left(\begin{array}{cc}1 & a_{i} \\ 0 & 1\end{array}\right)\left(\begin{array}{cc}1 & 0 \\ b_{i} & 1\end{array}\right)$. Conversely, any nonempty product of such factors is an element of $\mathrm{SL}_{2}(\mathbb{Z})$ with two distinct eigenvalues in $\mathbb{R}_{+}^{*}$.

We sketch a proof of this popular fact, mainly in order to introduce the cyclic word $\Omega$ associated to $\varphi$. The converse implication is easy (just check that the trace is larger than 2), so we focus on the direct statement.

Consider the upper half-plane model of the hyperbolic plane $\mathbb{H}^{2}$, endowed with the Farey tessellation $F$ (the ideal triangle $01 \infty$ iteratedly reflected in its sides). We identify $\mathrm{PSL}_{2}(\mathbb{R})$ with the group of isometries of $\mathbb{H}^{2}$ via the isomorphism $\Psi$ defined 
by

$$
\Psi\left(\begin{array}{ll}
a & b \\
c & d
\end{array}\right): z \mapsto \frac{d z+c}{b z+a} .
$$

(Under this slightly unusual convention, the slopes of the eigenvectors of $M$ are the fixed points of $\Psi(M)$, rather than their inverses as would normally be the case.) It is known that the group of orientation-preserving isometries of $\mathbb{t}^{2}$ preserving $F$ is thus identified with $\mathrm{PSL}_{2}(\mathbb{Z})$.

If $D$ is the oriented hyperbolic line running from the repulsive fixed point of $\pm \varphi$ to the attractive one, then $D$ crosses infinitely many Farey triangles $\left(\ldots t_{-1}, t_{0}, t_{1}, t_{2}, \ldots\right)$ of $F$. We can formally write down a bi-infinite word

$$
\Omega=\ldots L R R R L L R \ldots
$$

where the $k$-th letter is $R$ (resp. $L$ ) if $D$ exits $t_{k}$ to the Right (resp. Left) of where it enters. We will also say that $D$ makes a Right (resp. Left) at $t_{k}$. The word $\Omega$ contains at least one $R$ and one $L$, because the ends of $D$ are distinct. The image of $t_{0}$ under $\varphi$ is a certain $t_{m}(m>1)$, and $\Omega$ is periodic of period $m$.

Next, define the standard transvection matrices

$$
R:=\left(\begin{array}{ll}
1 & 1 \\
0 & 1
\end{array}\right) \text { and } L:=\left(\begin{array}{ll}
1 & 0 \\
1 & 1
\end{array}\right) .
$$

These are parabolic transformations of $\mathbb{M}^{2}$ whose respective fixed points are 0 and $\infty$. Let $M$ be any subword of $\Omega$ of length $m$ : we see $M$ as a product of standard transvection matrices, and therefore as an element of $\mathrm{SL}_{2}(\mathbb{Z})$. By studying the actions of $R$ and $L$ on $F$, it is then easy to see that $\varphi$ and $M$ are conjugates in $\operatorname{PSL}_{2}(\mathbb{Z})$, and therefore in $\mathrm{SL}_{2}(\mathbb{Z})$ since both have positive trace. This proves the existence statement for the $\left(a_{i}, b_{i}\right)$.

Uniqueness is checked as follows: on one hand, if $\varphi$ and $\varphi^{\prime}$ are conjugates, a certain element of $\operatorname{PSL}_{2}(\mathbb{Z})$ (preserving $F$ ) takes the axis of $\varphi$ to the axis of $\varphi^{\prime}$, so they define the same word $\Omega$ up to translation. On the other hand, looking at the actions of $R$ and $L$ on $\mathbb{H}^{2}$, one sees that a product of standard transvection matrices (as in the statement of Proposition 2.1) will define the word $\Omega=R^{a_{1}} L^{b_{1}} \ldots R^{a_{n}} L^{b_{n}}$, concatenated infinitely many times with itself.

In the language of Proposition 2.1, the sequence $\left(a_{1}, b_{1}, \ldots, a_{n}, b_{n}\right)$ can be shown to be (a positive power of) the period of the continued fraction expansion of the slope of the expanding eigenvector of $\varphi$. The word $\Omega$ will be seen either as infinite periodic, or as finite cyclic, depending on the context. 


\section{The canonical triangulation}

\subsection{Diagonal exchanges}

There is another well-known interpretation of the Farey tessellation $F$ of the hyperbolic plane $\mathbb{\boxplus}^{2}$. Under the canonical identification $H_{1}(T, \mathbb{Z}) \simeq \mathbb{Z}^{2}$, where $T$ is the punctured torus defined in the Introduction, each rational number in the boundary $\widehat{\mathbb{R}}=\mathbb{R} \cup\{\infty\}$ of $\mathbb{W}^{2}$ can be seen as a slope, ie a proper isotopy class of properly embedded lines in $T$, going from the puncture to itself. The action of $\mathrm{SL}_{2}(\mathbb{Z})$ on $\widehat{\mathbb{Q}}$ coincides with the action of the mapping class group $G$ of $T$ on rational slopes. The edges of the Farey tessellation $F$ connect exactly the pairs of rational numbers whose corresponding slopes, or curves in $T$, can be homotoped off each other (away from the puncture). The faces of $F$, having three edges, correspond exactly to the isotopy classes of ideal triangulations of $T$ : any such triangulation has one vertex (the puncture), three edges, and two triangles (which meet along each edge). As one crosses from a face of $F$ to one of its neighbors, exactly one vertex gets replaced, which in the corresponding triangulations of $T$ means that exactly one edge is changed. Inspection shows that the triangulation must be undergoing a diagonal exchange: erase one edge $e$, thus liberating a quadrilateral space $Q$ of which $e$ was a diagonal, then insert back the other diagonal.

\subsection{Tetrahedra}

As before, let $\varphi$ be an element of $\mathrm{SL}_{2}(\mathbb{Z})$ with two distinct eigenvalues in $\mathbb{R}_{+}^{*}$. In the proof of Proposition 2.1, we introduced the triangles $t_{0}, t_{1}, \ldots$ crossed by the axis $D$ of $\varphi$. In view of the above, this yields a nonbacktracking path of diagonal exchanges between some triangulation (associated to $t_{0}$ ) and its pushforward by $\varphi$ (associated to $\left.t_{m}\right)$.

More precisely, when the oriented line $D$ crosses an edge $e$ of the Farey tessellation, $e$ comes with a transverse orientation. So we can define the top (resp. bottom) triangulation $\tau_{+}(e)$ (resp. $\left.\tau_{-}(e)\right)$ of the punctured torus $T$ as being the one associated with the Farey triangle crossed just after (resp. before) the edge $e$. A diagonal exchange separates the triangulations $\tau_{-}(e)$ and $\tau_{+}(e)$. An ideal tetrahedron is by definition a space diffeomorphic to an ideal hyperbolic tetrahedron (topologically it is a compact tetrahedron with its vertices removed). We can immerse such an ideal tetrahedron $\Delta(e)$ in $T \times \mathbb{R}$ : the boundary of the immersed $\Delta(e)$ is made up of two pleated surfaces (top and bottom) homotopic to $T$ and triangulated according to $\tau_{+}(e)$ and $\tau_{-}(e)$ respectively (Figure 1). The immersion is an embedding on the interior of $\Delta(e)$, but two pairs of opposite edges undergo identifications. 


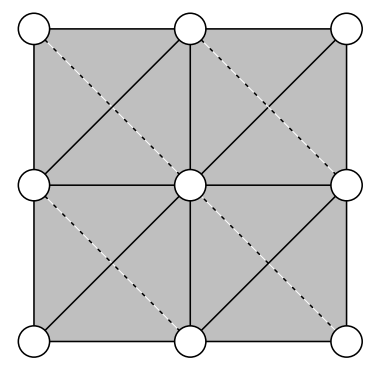

Figure 1: Four copies of $\Delta(e)$ in the cover $\left(\mathbb{R}^{2} \backslash \mathbb{Z}^{2}\right) \times \mathbb{R}$ of $T \times \mathbb{R}$

Next, if $D$ crosses the Farey edges $e_{i}, e_{i+1}, \ldots$, we can glue the top of the tetrahedron $\Delta_{i}:=\Delta\left(e_{i}\right)$ onto the bottom of $\Delta_{i+1}$ in $T \times \mathbb{R}$, because $\tau_{+}\left(e_{i}\right)=\tau_{-}\left(e_{i+1}\right)$. We thus get a bi-infinite stack of tetrahedra $\left(\Delta_{i}\right)_{i \in \mathbb{Z}}$. For any nonnegative $N$ the space $U_{N}:=\bigcup_{i=-N}^{N} \Delta_{i}$ is a strong deformation retraction of $T \times \mathbb{R}$. For $N$ large enough, $U_{N}$ is homeomorphic to $T \times[0,1]$ : the only way this can fail is if all the $\Delta_{i}$ for $-N \leq i \leq N$ have a common edge; but any edge of any tetrahedron $\Delta_{j}$ is shared by only finitely many other (consecutive) $\Delta_{i}$ 's, because for any Farey vertex $v$, only finitely many of the Farey edges $e_{i}$ bound triangles with $v$ as a vertex (and these $e_{i}$ are consecutive). Therefore, the space $U=\bigcup_{i \in \mathbb{Z}} \Delta_{i}$ is homeomorphic to $T \times \mathbb{R}$. If $m$ is the period of the word $\Omega$, there is an orientation-preserving homeomorphism $\Phi$ of $U$, acting like $[\varphi]$ on the $T$-factor, that sends $\Delta_{i}$ to $\Delta_{i+m}$ for all $i$. The quotient $U / \Phi$ is a manifold (homeomorphic to) $V_{\varphi}$, naturally triangulated into $m$ ideal tetrahedra.

Figure 2 also shows a way to interpret the standard transvection matrices $R$ and $L$ directly as adjunctions of new tetrahedra (by performing diagonal exchanges on the top faces). Similarly, to topologically triangulate a general pseudo-Anosov surface bundle, we can always go by diagonal exchanges from some triangulation (of the surface) to its pushforward by the monodromy map, an idea usually credited to Casson.

\section{Combinatorics of the torus at infinity}

The manifold $V_{\varphi}$ is naturally homeomorphic to the interior of a compact manifold with boundary, denoted by $\bar{V}_{\varphi}$ and defined as a bundle over the circle with fiber $T-\delta$, where $\delta$ is a regular neighborhood of the puncture.

The torus at infinity of the manifold $V_{\varphi}$ is the boundary of $\bar{V}_{\varphi}$, namely a topological torus. The links of the vertices of the tetrahedra $\Delta_{i}$ provide a tessellation $\mathcal{A}$ of the torus at infinity into topological triangles. In this section we investigate the combinatorics of $\mathcal{A}$. 


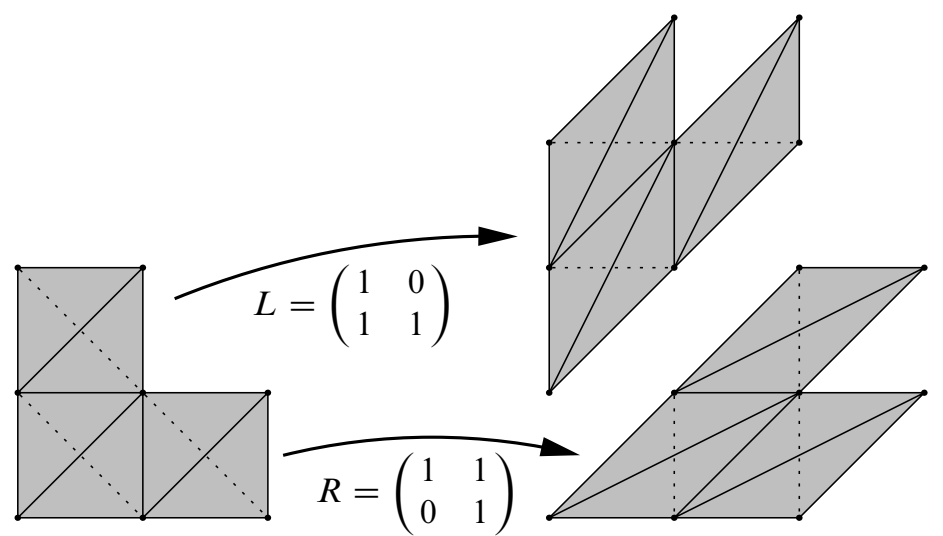

Figure 2

Each vertex of $\mathcal{A}$ corresponds to an edge of $V_{\varphi}$ shared by a few consecutive tetrahedra $\Delta_{i}$. This edge in turn corresponds to a Farey vertex shared by a few consecutive Farey triangles. The union of all the Farey triangles adjacent to a given vertex $v$ forms a fan. If $v$ arises as a vertex of triangles visited by the oriented line $D$, one of the following two things must happen right after $D$ enters the fan: either $D$ makes a Right, then a number of Lefts (possibly 0), then a Right and leaves the fan; or the same is true, exchanging Right and Left.

Therefore, the vertices of $\mathcal{A}$ correspond exactly to the subwords of $\Omega$ of the form $R L^{*} R$ or $L R^{*} L$ (where $* \geq 0$ ). Each such subword actually corresponds to two vertices of $\mathcal{A}$, because the edges of the tetrahedra $\Delta_{i}$ have two ends.

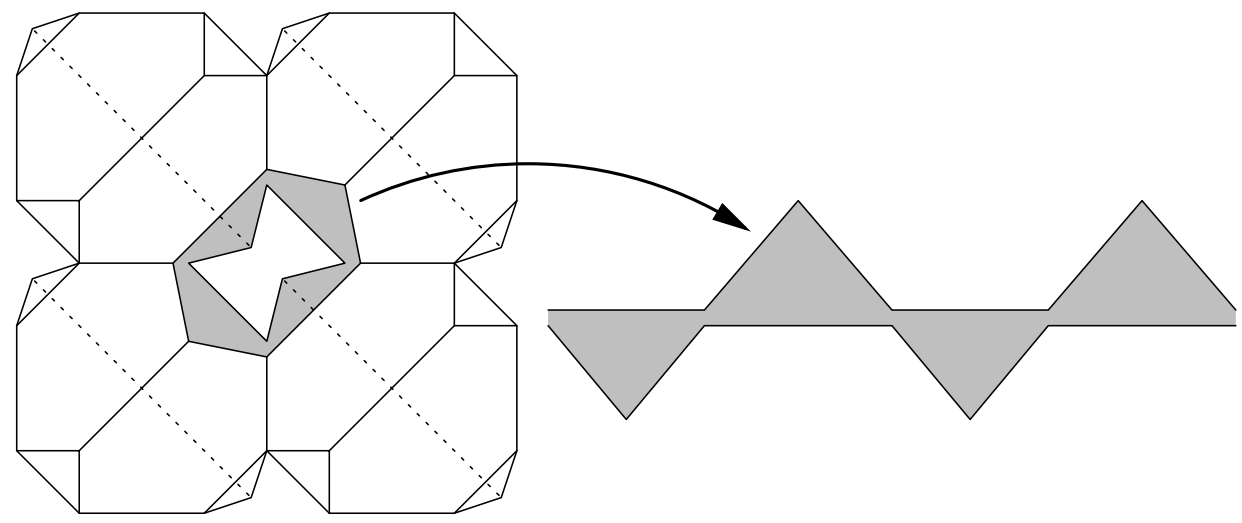

Figure 3: The link of the puncture 
Moreover, each tetrahedron $\Delta_{i}$, having four vertices, contributes exactly four triangles to $\mathcal{A}$. By looking at a vertex (puncture) of the cover $\left(\mathbb{R}^{2} \backslash \mathbb{Z}^{2}\right) \times \mathbb{R}$ of $T \times \mathbb{R}$ with embedded $\Delta_{i}$, one checks (Figure 3 ) that each of the four triangles has exactly one vertex not shared with any of the other three: we call this vertex the apex and the opposite edge the base. The four bases form a broken line of four segments which is a closed curve running around the puncture, and the four apices point alternatively up and down in the $\mathbb{R}$-factor. Such chains of four triangles must be stacked on top of one another while respecting the previously described combinatorics of the vertices. The result is shown in Figure 4, where the underlying word $\Omega$ was chosen to be $\ldots R^{4} L^{4} R^{4} L^{4} \ldots$ (read from bottom to top). A few remarks are in order.

First remark We labeled by $x_{i}, y_{i}, z_{i}$ the angular sectors of the triangles corresponding to the tetrahedron $\Delta_{i}$ (in Figure 4, the subscript $i$ is replaced by a number at the center of the triangle, omitted after the first few levels). Each angular sector corresponds to a (topological) dihedral angle of $\Delta_{i}$. Opposite dihedral angles are equal in ideal hyperbolic tetrahedra: this is why three different labels per level are enough (instead of six, the total number of edges in a tetrahedron).

Second remark The design in Figure 4 of the vertices of the torus at infinity, represented as being "opened up", is intended to emphasize the layered structure of the picture (each layer corresponds to one tetrahedron $\Delta_{i}$ ).

Third remark Proving Theorem 1.1 by the method outlined in the Introduction amounts to realizing Figure 4 geometrically in the plane by Euclidean triangles, making same-layer angles with identical labels equal (Lemma 6.2 will make this statement more precise).

Fourth remark The convention is that the pair of edges of $\Delta_{i}$ that does not get identified into one edge is labeled $z_{i}$ : so $z_{i}$ is the label at the apex (in the sense of Figure 3). Equivalently, if a tetrahedron is seen as a diagonal exchange, $z_{i}$ is the label common to the appearing and disappearing edges. The other edge pairs are labeled in such a way that in each triangle the letters $x, y, z$ appear clockwise in that order. Therefore, if $e_{i}$ is a Farey edge and $p$ (resp. $q$ ) its right (resp. left) end for the transverse orientation, the dihedral angle of the tetrahedron $\Delta_{i}$ at the edge of slope $p$ (resp. $q$ ) is $x_{i}\left(\right.$ resp. $\left.y_{i}\right)$.

Fifth remark The tetrahedra $\Delta_{i}$ are naturally indexed in $\mathbb{Z} / m \mathbb{Z}$. The letters $R$ and $L$ live naturally on the pleated surfaces, or between the tetrahedra $\Delta_{i}$. In Figure 4 and henceforth, the $i$-th layer is colored in grey if and only if the letters just before and 


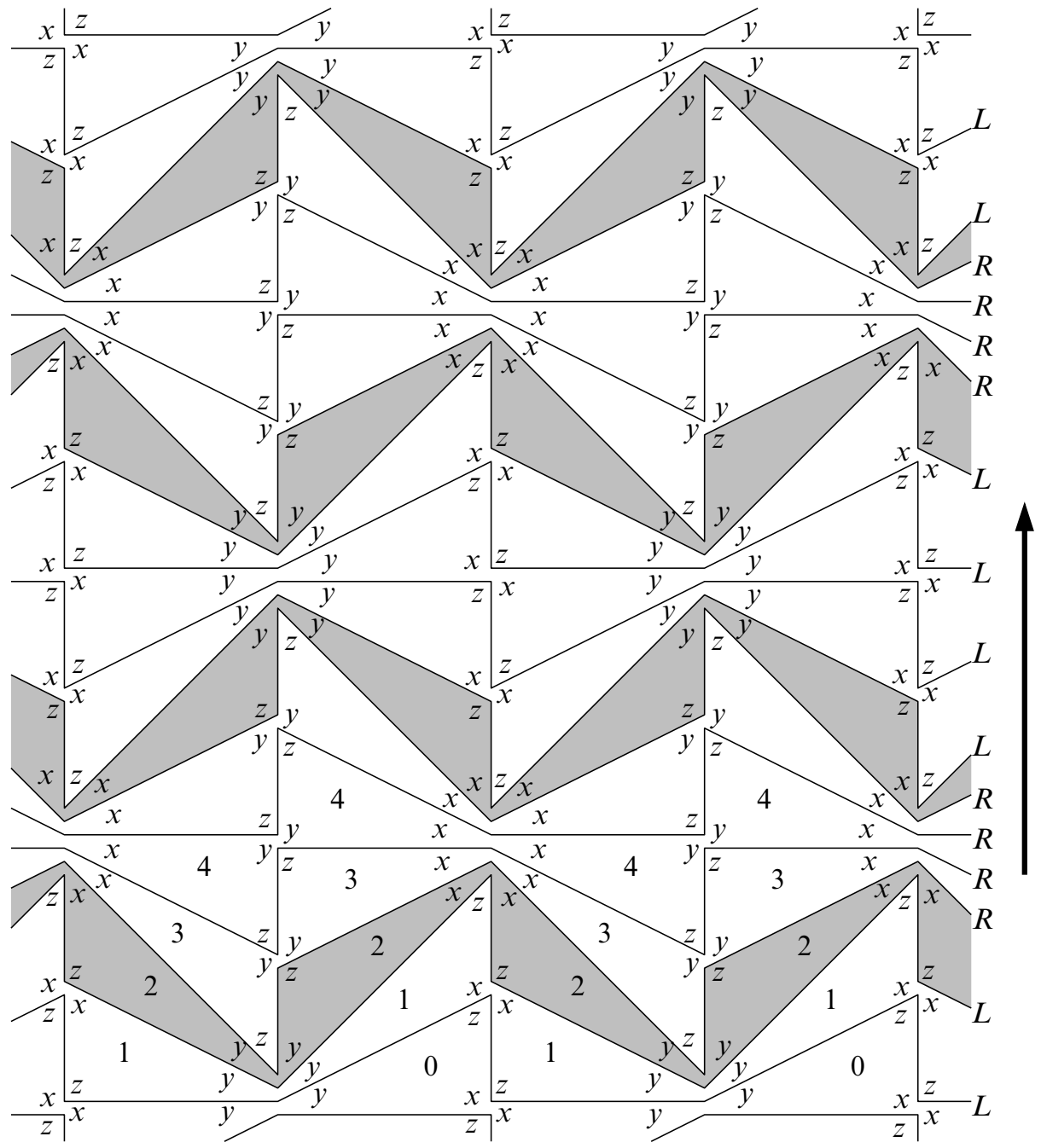

Figure 4: The tessellation $\mathcal{A}$

just after $\Delta_{i}$ are different (here $i=2,6, \ldots$ ). Such indices $i$ are called hinge indices, because they are at the hinge between two nonempty subwords $R^{p}$ and $L^{q}$. Hinge tetrahedra (the associated $\Delta_{i}$ ) will play an important role later on.

Sixth remark While the fundamental domain of Figure 4 is supposed to have a horizontal length of four triangles (see Figure 3), we notice a horizontal period of length only two. This corresponds to the "hyperelliptic" involution of the once-punctured 
fiber torus (rotation of $180^{\circ}$ around the puncture, central in $\mathrm{SL}_{2}(\mathbb{Z})$ and therefore well-defined on $V_{\varphi}$ ). This will simplify many of our computations.

Seventh remark The valence of a vertex $s$ corresponding to a subword $R L^{n} R$ or $L R^{n} L$ of $\Omega$ (where $n \geq 0$ ) is $2 n+4$. This is because exactly $n+2$ Farey triangles are adjacent to the corresponding rational $v_{s}$; each such Farey triangle defines a triangulated surface (with an edge of slope $v_{s}$ ), and each such surface contributes exactly two segments issuing from $s$ in $\mathcal{A}$.

\section{Finding positive angles}

The tetrahedra $\Delta_{i}$ and $\Delta_{i+1}$ have two common triangular faces whose union forms a pleated punctured torus $\Sigma$ properly isotopic to $T \times\{*\}$ in $T \times \mathbb{R}$. Moreover, $\Sigma$ receives a transverse "upward" orientation from the $\mathbb{R}$-factor. Suppose all tetrahedra $\Delta_{i}$ are endowed with dihedral angles. Let $e$ be an edge of $\Sigma$ : if the sum of all dihedral angles at $e$ below $\Sigma$ is $\pi+\alpha$, we call $\alpha$ the pleating angle of $\Sigma$ at $e$.

In this section we find positive dihedral angles for the ideal tetrahedra $\Delta_{i}$. More precisely, we describe the convex space $\Pi$ of positive angles $x_{i}, y_{i}, z_{i}$ for the $\Delta_{i}$ such that:

$$
\left\{\begin{aligned}
\mathrm{i}- & \text { For each } i \text { in } \mathbb{Z} / m \mathbb{Z} \text { one has } x_{i}+y_{i}+z_{i}=\pi ; \\
\mathrm{ii}- & \text { The dihedral angles around any edge add up to } 2 \pi ; \\
\text { iii }- & \text { For each } i \text { in } \mathbb{Z} / m \mathbb{Z} \text {, the three pleating angles of the pleated } \\
& \text { punctured torus between } \Delta_{i} \text { and } \Delta_{i+1} \text { add up to } 0 .
\end{aligned}\right.
$$

Condition (1)-ii is necessary, though not sufficient, for a hyperbolic structure at the edges; Condition (1)-iii is necessary, though not sufficient, to make the loop around the puncture of $T$ a parabolic isometry of $\mathbb{H}^{3}$ (see Sublemma 6.4). This "cusp condition" (1)-iii restricts the space of angle structures, but will make it a little easier to describe.

Recall the line $D$ that runs from the repulsive fixed point $q^{-}$to the attractive fixed point $q^{+}$of $\varphi$ across the Farey triangulation. If the tetrahedron $\Delta_{i}$, corresponding to the Farey edge $e_{i}$, realizes a diagonal exchange that erases an edge $\varepsilon^{\prime}$ and replaces it with $\varepsilon$, we denote by $z_{i}$ the interior dihedral angle of $\Delta_{i}$ at $\varepsilon$ and $\varepsilon^{\prime}$ by the fourth remark following Figure 4. Observe that the slope of $\varepsilon$ (resp. $\varepsilon^{\prime}$ ) is the rational located opposite $e_{i}$ in the Farey diagram, on the side of $q^{+}$(resp. $q^{-}$). We define the half pleating angle $w_{i}$ by $\pi-2 w_{i}=z_{i}$.

Thus, if (1)-ii and (1)-iii are to be satisfied, the pleating angles of the pleated punctured torus $\Sigma$ living between $\Delta_{i}$ and $\Delta_{i+1}$ must be

$$
-2 w_{i}, \quad 2 w_{i+1} \text { and } 2 w_{i}-2 w_{i+1} .
$$


(Observe the signs: by our definition, angles pointing upwards, like the "new" edge of $\Delta_{i}$, are negative pleatings, while angles pointing downwards, like the "old" edge of $\Delta_{i+1}$, are positive ones.) We write the numbers (2) in the corners of the corresponding Farey triangle (top of Figure 5), distinguishing the cases $L$ and $R$, and we repeat this for all indices $i$.
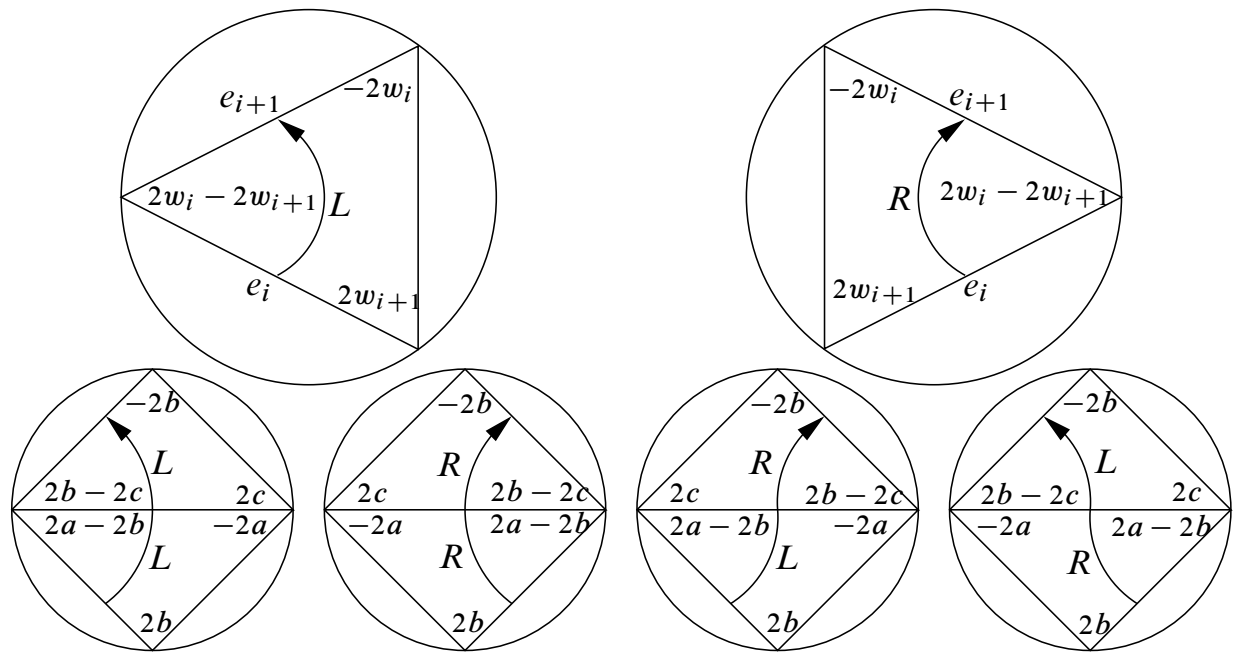

Figure 5: On the bottom row in each case, $e_{i}$ is the horizontal edge

In fact, the values of the $w_{i}$ will also determine the $x_{i}$ and $y_{i}$. To see this, we must write down the pleating angles of two pleated surfaces, living above and below the tetrahedron $\Delta_{i}$. For notational convenience, write

$$
\left(w_{i-1}, w_{i}, w_{i+1}\right)=:(a, b, c) \text {. }
$$

By the fourth remark following Figure 4, the quantity $2 x_{i}$ (resp. $2 y_{i}$ ) is the difference between the numbers written just above and just below the right (resp. left) end of $e_{i}$ in Figure 5 (the factor 2 comes from the fact that the two edges of $\Delta_{i}$ with angle $x_{i}$ (resp. $\left.y_{i}\right)$ are identified). By computing differences between the pleating angles given in Figure 5 (bottom), we find the formulae in Table 1 for $x_{i}, y_{i}, z_{i}$ (depending on the letters $\Omega_{i}^{-}$and $\Omega_{i}^{+}$, each equal to $R$ or $L$, living respectively just before and just after the index $i$ ). 


\begin{tabular}{c|c|c|c|c}
$\Omega_{i}^{-}, \Omega_{i}^{+}$ & $L L$ & $R R$ & $L R$ & $R L$ \\
\hline$x_{i}$ & $a+c$ & $-a+2 b-c$ & $a+b-c$ & $-a+b+c$ \\
$y_{i}$ & $-a+2 b-c$ & $a+c$ & $-a+b+c$ & $a+b-c$ \\
$z_{i}$ & $\pi-2 b$ & $\pi-2 b$ & $\pi-2 b$ & $\pi-2 b$
\end{tabular}

Table 1

Condition (1)-i can be checked immediately, while (1)-ii-iii are true by construction. From Table 1, the conditions for all angles $x_{i}, y_{i}, z_{i}$ to be positive are that:

$$
\left\{\begin{aligned}
\mathrm{i}- & \text { For all } i \text { one has } 0<w_{i}<\pi / 2 \\
\mathrm{ii}- & \text { If } i \text { is not a hinge index (first two cases), } 2 w_{i}>w_{i+1}+w_{i-1} \\
\text { iii }- & \text { If } i \text { is a hinge index (last two cases), }\left|w_{i+1}-w_{i-1}\right|<w_{i}
\end{aligned}\right.
$$

We call (3)-i the range condition, (3)-ii the concavity condition, and (3)-iii the hinge

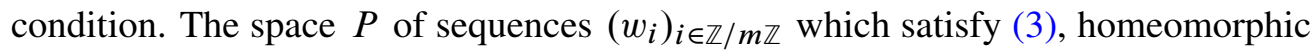
to the solution space $\Pi$ of (1), is clearly an open, convex polyhedron of compact closure in $\mathbb{R}^{m}$. Moreover, $P$ is nonempty: to exhibit a sequence $\left(w_{i}\right)$ in $P$, set $w_{j}=\pi / 3$ when $j$ is a hinge index, and complete the gaps between consecutive hinge indices $j<k$ with strictly concave subsequences taking their values in $[\pi / 3, \pi / 2)$, eg $w_{i}=\pi / 3+\frac{(i-j)(k-i)}{(k-j)^{2}}$ for $j \leq i \leq k$ (indices are of course seen in $\mathbb{Z}$ for the evaluation). Figure 6 shows the typical graph of a sequence $\left(w_{i}\right)$ that satisfies all conditions of (3). Finally, note that the formulae of Table 1 are still valid when $\Omega$ is reduced to $R L$ or $L R$ (the letters $a$ and $c$ are just two names for the same parameter then).

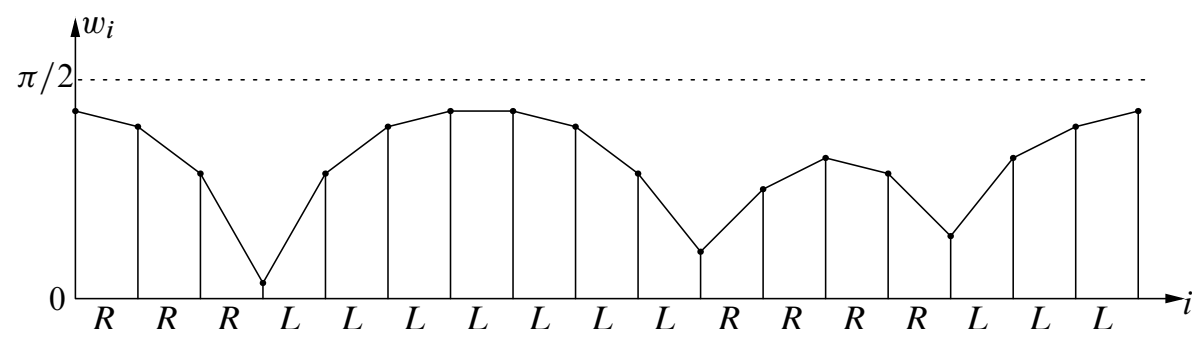

Figure 6 


\section{Hyperbolic volume}

Our goal for the remainder of the paper is to find a point $\left(w_{i}\right)$ of $P$ where the tetrahedra fit together so as to yield a complete hyperbolic structure on $V_{\varphi}$. This section is devoted to checking that this is the same as finding in $P$ a critical point of the total hyperbolic volume, an approach pioneered by Rivin [22]. A few facts concerning the volume of ideal tetrahedra will be needed.

\subsection{Volume of an ideal tetrahedron}

The volume of a hyperbolic tetrahedron whose dihedral angles are $x, y, z>0$ is

(4) $\mathcal{V}(x, y, z)=-\int_{0}^{x} \log (2 \sin u) d u-\int_{0}^{y} \log (2 \sin u) d u-\int_{0}^{z} \log (2 \sin u) d u$

(we refer to Milnor [17] for a proof). Since $\int_{0}^{\pi} \log (2 \sin u) d u=0$, Equation (4) easily implies the following proposition.

Proposition 6.1 The function $\mathcal{V}$ can be continuously extended by 0 to all nonnegative triples $(x, y, z)$ such that $x+y+z=\pi$. If $\frac{d}{d t}\left(x_{t}, y_{t}, z_{t}\right)=(X, Y, Z)$ then

$$
\exp \left(\frac{-d}{d t} \mathcal{V}\left(x_{t}, y_{t}, z_{t}\right)\right)=\sin ^{X} x_{t} \sin ^{Y} y_{t} \sin ^{Z} z_{t} \text {. }
$$

Proof Straightforward. We will always apply this formula exactly in the form it is stated, because it will usually make the right hand side simplest.

\subsection{Critical volume and trivial holonomy}

Lemma 6.2 (Rivin, Chan-Hodgson) On the open affine polyhedron $P$ of sequences $\left(w_{i}\right)$ satisfying (3), define the volume functional $\mathcal{V}$ as the sum of the hyperbolic volumes of tetrahedra $\Delta_{i}$ with dihedral angles $x_{i}, y_{i}, z_{i}$, as given by Table 1 . Then $\left(w_{i}\right)$ is a critical point for $\mathcal{V}$ in $P$ if and only if the gluing of the tetrahedra $\Delta_{i}$ defines a complete finite-volume hyperbolic structure on the punctured torus bundle $V_{\varphi}$.

Proof This now standard fact holds for general ideal triangulations as well (see for example Chan [9] or Rivin [22]). The following proof, however, is deliberately specific to the example at hand. This will enable us to introduce a few objects and relationships that will be useful in the sequel. Conversely, the main idea of the present proof (associate to each edge of $V_{\varphi}$ an explicit deformation in the space of angle structures) can be used to prove the general case of Rivin's theorem. 
First we assume $\left(w_{i}\right)$ is critical. Let $B$ be the torus at infinity of $V_{\varphi}$ with the vertices of the tessellation $\mathcal{A}$ removed. Let $\sigma$ be the hyperelliptic involution of the fiber $T$, so $\sigma$ acts as a translation on $B$. Define $B^{\prime}=B / \sigma$ and $\mathcal{A}^{\prime}=\mathcal{A} / \sigma$. Let $t_{0}$ be a triangle of $\mathcal{A}^{\prime}, \epsilon_{0}$ an oriented edge of $t_{0}$ and $x_{0}$ an interior point of $t_{0}$. The group of orientation-preserving similarities of the Euclidean plane $\mathbb{C}$ is $\mathbb{C}^{*} \ltimes \mathbb{C}$.

Definition 6.3 For a given $\left(w_{i}\right)$ in $P$, the holonomy function is the representation

$$
\rho: \pi_{1}\left(B^{\prime}, x_{0}\right) \rightarrow \mathbb{C}^{*} \ltimes \mathbb{C}
$$

defined as follows. Given an element $\alpha$ of $\pi_{1}\left(B^{\prime}, x_{0}\right)$, view $\alpha$ as a cyclic sequence of triangles $t_{0}, t_{1}, \ldots, t_{s}=t_{0}$ of $\mathcal{A}^{\prime}$, such that any two consecutive $t_{i}$ 's share an edge. Then, draw an oriented copy $\tau_{0}$ of $t_{0}$ in the plane $\mathbb{C}$, with angles specified by $\left(w_{i}\right)$, by making the image of the oriented edge $\epsilon_{0}$ coincide with $(0,1)$. Sharing an edge with $\tau_{0}$, draw a copy $\tau_{1}$ of $t_{1}$, also with angles specified by $\left(w_{i}\right)$. Then draw a copy $\tau_{2}$ of $t_{2}$ adjacent to $\tau_{1}$, etc. By definition, $\rho(\alpha)$ is the orientation-preserving similarity mapping the copy of the oriented edge $\epsilon_{0}$ in $\tau_{0}$ to the copy of $\epsilon_{0}$ in $\tau_{s}$. The reduced holonomy function $\psi: \pi_{1}\left(B^{\prime}, x_{0}\right) \rightarrow \mathbb{C}^{*}$ is defined as the projection of $\rho$ on the first factor.

It is a simple exercise to check that $\rho$ is well-defined, and is a representation (the concatenation rule being that $\alpha \beta$ denotes the path $\alpha$ followed by the path $\beta$ ). Note that $\psi$, having a commutative target, factors through a representation $\psi: H_{1}\left(B^{\prime}, \mathbb{Z}\right) \rightarrow \mathbb{C}^{*}$.

Sublemma 6.4 Let $\alpha$ be an element of $H_{1}\left(B^{\prime}, \mathbb{Z}\right)$ represented by a curve around a 4-valent vertex of $\mathcal{A}^{\prime}$, and let $\beta$ be represented by a curve that follows a "grey" (hinge) level in $\mathcal{A}^{\prime}$ (Figure 7). If $\left(w_{i}\right)$ is critical for the volume functional $\mathcal{V}$, then $\psi(\alpha)=\psi(\beta)=1$.

Proof We already know that $\psi(\alpha), \psi(\beta)$ belong to $\mathbb{R}_{+}^{*}$, since the angle conditions (1) defining $P$ impose oriented parallelism. It remains to prove that $|\psi(\alpha)|=|\psi(\beta)|=1$. At a critical point, the partial derivatives of $\mathcal{V}$ with respect to any of the $w_{i}$ must vanish. Between and near two identical letters, say $R$ and $R$, according to Table 1 , the angles are given by the table

\begin{tabular}{c|ccc}
$\Omega$ & & $R$ & \multicolumn{2}{r}{$R$} \\
$i$ & 0 & 1 & 2 \\
$w_{i}$ & $a$ & $b$ & $c$ \\
\hline$x_{i}$ & $\xi-b$ & $-a+2 b-c$ & $-b+\xi^{\prime}$ \\
$y_{i}$ & $\eta+b$ & $a+c$ & $b+\eta^{\prime}$ \\
$z_{i}$ & $\pi-2 a$ & $\pi-2 b$ & $\pi-2 c$
\end{tabular}



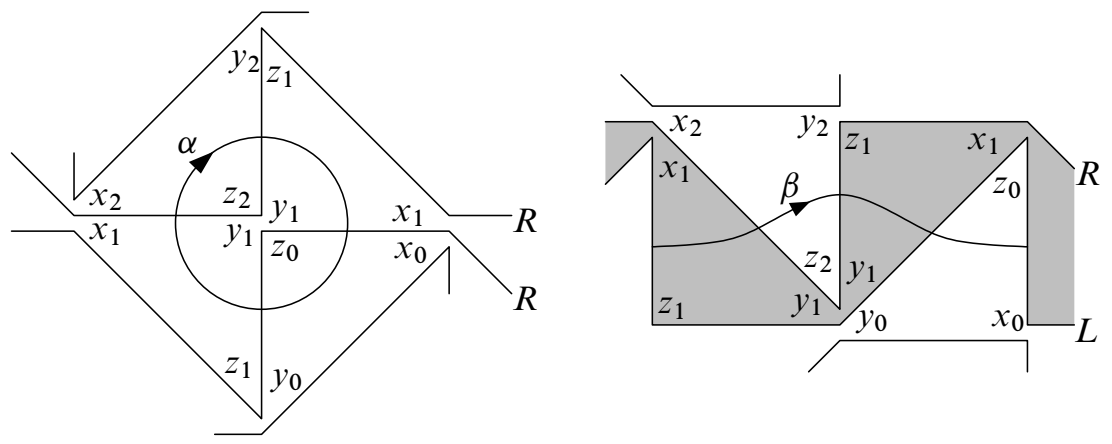

Figure 7

(the exact expression of $\xi, \xi^{\prime}, \eta, \eta^{\prime}$ depends on the letters before and after $R R$, but only the $b$-contribution matters here). Using Proposition 6.1, criticality of $\mathcal{V}$ implies

$$
1=\exp \frac{-\partial \mathcal{V}}{\partial b}=\frac{\sin y_{0} \sin ^{2} x_{1} \sin y_{2}}{\sin x_{0} \sin ^{2} z_{1} \sin x_{2}} .
$$

Using the fact that edge lengths in a triangle are proportional to angle sines, it follows that the edge lengths in Figure 7 (left) around the central vertex fit nicely together. So $|\psi(\alpha)|=1$. The case of a subword $L L$ is treated similarly, which takes care of all 4-valent vertices of the tessellation $\mathcal{A}^{\prime}$.

Near a hinge between two different letters, say $L$ followed by $R$, the angles are

\begin{tabular}{c|ccc}
$\Omega$ & & $L$ & \multicolumn{2}{c}{$R$} \\
$i$ & 0 & 1 & 2 \\
$w_{i}$ & $a$ & $b$ & $c$ \\
\hline$x_{i}$ & $\xi+b$ & $a+b-c$ & $-b+\xi^{\prime}$ \\
$y_{i}$ & $\eta-b$ & $-a+b+c$ & $b+\eta^{\prime}$ \\
$z_{i}$ & $\pi-2 a$ & $\pi-2 b$ & $\pi-2 c$
\end{tabular}

This time, criticality implies

$$
1=\exp \frac{-\partial \mathcal{V}}{\partial b}=\frac{\sin x_{0} \sin y_{1} \sin x_{1} \sin y_{2}}{\sin y_{0} \sin ^{2} z_{1} \sin x_{2}} .
$$

As shown in Figure 7 (right) and by the same trigonometric argument, this means that the first and last edges crossed by $\beta$ have the same length. So $\psi(\beta)=1$. (If $\Omega$ is reduced to $L R$, the computation is formally the same, identifying indices 0 and 2.) The case of a subword $R L$ is similar. Sublemma 6.4 is proved. 
Now let $\alpha$ be an element of $\pi_{1}\left(B^{\prime}, x_{0}\right)$ that is conjugated to a simple loop around a 4-valent vertex of $\mathcal{A}^{\prime}$. By Sublemma 6.4 (and an easy conjugation argument), $\rho(\alpha)$ is a translation. Moreover, $\rho(\alpha)$ fixes the vertex around which $\alpha$ revolves, so $\rho(\alpha)=1$, the identity of the Euclidean plane.

Sublemma 6.5 Let $U$ be the quotient of the torus at infinity of $V_{\varphi}$ by the action of the hyperelliptic involution $\sigma$ of the fiber, so that $B^{\prime} \subset U$. Suppose $\left(w_{i}\right)$ is critical for $\mathcal{V}$. Then the representation $\rho: \pi_{1}\left(B^{\prime}, x_{0}\right) \rightarrow \mathbb{C}^{*} \ltimes \mathbb{C}$ descends to a representation $\rho_{U}: \pi_{1}\left(U, x_{0}\right) \rightarrow \mathbb{C}^{*} \ltimes \mathbb{C}$ whose first projection $\psi_{U}: \pi_{1}\left(U, x_{0}\right) \rightarrow \mathbb{C}^{*}$ is trivial.

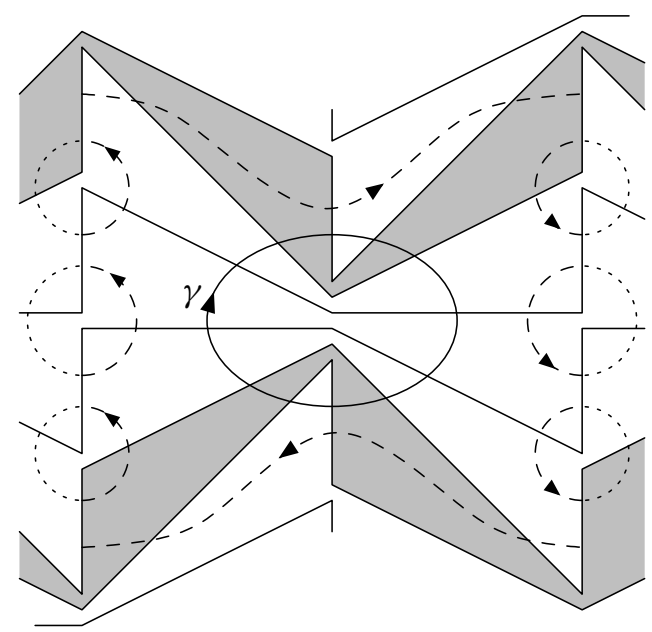

Figure 8

Proof To see that $\rho_{U}$ is well-defined, we only need to check that, if $\gamma$ is (conjugated to) a loop around a vertex $v$ of $\mathcal{A}^{\prime}$, then $\rho(\gamma)=1$. If $v$ has valence 4 , it has already been done. If not, by the argument preceding Sublemma 6.5, it is sufficient to check that $\psi([\gamma])=1$, where $[\gamma]$ denotes the homology class of $\gamma$. But in $H_{1}\left(B^{\prime}, \mathbb{Z}\right)$, the element $[\gamma]$ is a sum of loops around 4-valent vertices and curves following "grey" levels (see Figure 8: the vertical edges of $B^{\prime}$ on the two sides of the picture are identified, and the curves crossing these edges undergo a "split-and-paste" process to yield $\gamma$ ). So by Sublemma $6.4, \psi([\gamma])=1$; therefore $\rho_{U}$ is well-defined. Moreover, if $\beta$ is a curve following a "grey" level, Sublemma 6.4 tells that $\rho_{U}(\beta)=\rho(\beta)$ is a nonidentical Euclidean translation. The value of $\rho_{U}$ on another generator of $\pi_{1}(U)$ (which is abelian) must commute with $\rho_{U}(\beta)$, and therefore be a translation too. So $\rho_{U}$ has its image contained in $\{1\} \ltimes \mathbb{C}$ and $\psi_{U}=1$, completing the proof. 
By assigning length 1 , for example, to the reference edge $\epsilon_{0}$ of $\mathcal{A}^{\prime}$, a critical point $\left(w_{i}\right)$ of the volume functional thus defines the lengths of all other edges of $\mathcal{A}^{\prime}$ in a coherent way. This yields a complete Euclidean metric $g$ on $U$. The universal cover $\widetilde{U}$ of $U$ thus embeds into $\mathbb{C}$ (the embedding, also called the developing map of the local Euclidean structure, can only be injective, because the $\widetilde{g}$-geodesic joining two distinct points of $\widetilde{U}$ is sent to a geodesic of $\mathbb{C}$ ); so there is an isometry $\widetilde{U} \simeq \mathbb{C}$. The metric $g$ lifts from $U$ to the torus at infinity of $V_{\varphi}$ and its tessellation $\mathcal{A}$, producing a geometric realization of $\mathcal{A}$ and of Figure 4 in $\mathbb{C}$ (Euclidean plane tiling). Above each triangle of the universal cover of $\mathcal{A}$ now sits one ideal tetrahedron with vertex at infinity: the tetrahedron is the hyperbolic convex hull of $\infty$ and the vertices of the triangle. Note that these tetrahedra fill $\mathbb{M}^{3}$ completely above a certain height.

To make sure that the pasted metric on the union $V=V_{\varphi}$ of all ideal tetrahedra is now complete, assume a geodesic $\gamma(t)$ in $V$ hits infinity at time $T<\infty$. If $K \subset V$ is compact, ie has a compact intersection with each tetrahedron $\Delta_{i}$, then $\gamma$ must eventually exit $K$ (if not, the $\gamma(T-1 / n)$ accumulate at some point $p$ of some tetrahedron, but centered at $p$ there is a small embedded hyperbolic ball: absurd). So for $t$ sufficiently close to $T$, there is a lift of $\gamma(t)$ arbitrarily high above the tessellation $\mathcal{A}$ (embedded in $\mathbb{C}$ in the upper half space model). But at sufficiently great height, the tetrahedra above $\mathcal{A}$ fill $\mathbb{M}^{3}$ completely, so geodesics are defined for long times (eg times larger than 1): a contradiction. The first implication of Lemma 6.2 is proved.

To prove the converse, it is enough to show that if the gluing of the tetrahedra yields a complete hyperbolic metric, then the gluing of their vertex links yields a geometric realization of $\mathcal{A}$, ie of Figure 4 (checking $\partial \mathcal{V} / \partial w_{i}=0$ then amounts to a rerun of the two computations in Sublemma 6.4, distinguishing whether $i$ is a hinge index or not). But the latter is clear: given a complete hyperbolic metric, consider a triangulated universal cover $\mathbb{M}^{3} \rightarrow V_{\varphi}$ and send (a lift of) the cusp to infinity in the upper half space model. It is a classic argument that deck transformations of $\mathbb{U}^{3}$ which fix infinity must be parabolic, so the link of infinity has two translational periods and provides a Euclidean realization of $\mathcal{A}$ (and of Figure 4).

\subsection{Behavior of the volume functional}

As a consequence of Lemma 6.2, to prove Theorem 1.1 we only need to find a critical point of the volume functional $\mathcal{V}$ in the open polyhedron $P$ of cyclic sequences $\left(w_{i}\right)$ satisfying the conditions (3). A few more facts about the volume of ideal hyperbolic tetrahedra will be needed.

By Proposition 6.1, the volume functional $\mathcal{V}$ continuously extends to the (compact) closure $\bar{P}$ of the polyhedron $P$ (the space $\bar{P}$ is defined by turning the conditions 
(3) to weak inequalities, or taking the limits in $\mathbb{R}^{m}$ of sequences of $P$ ). Then $\mathcal{V}$ has well-defined extrema on $\bar{P}$, which are automatically critical if they belong to $P$. Because of the following proposition, the only possibility for a critical point is to be an absolute maximum.

Proposition 6.6 The volume of an ideal tetrahedron is a concave function of its dihedral angles.

Proof This follows from Proposition 6.1, whose notations we use again: $x_{t}, y_{t}, z_{t}$ are the dihedral angles. By symmetry we may assume $x_{0}, y_{0} \leq \pi / 2$. Assume further that $x_{t}, y_{t}, z_{t}$ are affine functions of $t$ with first-degree coefficients $X, Y, Z$. Proposition 6.1 implies $-d \mathcal{V} / d t=X \log \sin x_{t}+Y \log \sin y_{t}+Z \log \sin z_{t}$, and by differentiating we obtain

$$
\begin{aligned}
-d^{2} \mathcal{V} /\left.d t^{2}\right|_{t=0} & =X^{2} \cot x_{0}+Y^{2} \cot y_{0}+Z^{2} \cot z_{0} \\
& =X^{2} \cot x_{0}+Y^{2} \cot y_{0}+(X+Y)^{2} \frac{1-\cot x_{0} \cot y_{0}}{\cot x_{0}+\cot y_{0}} \\
& =\frac{(X+Y)^{2}+\left(X \cot x_{0}-Y \cot y_{0}\right)^{2}}{\cot x_{0}+\cot y_{0}} \geq 0
\end{aligned}
$$

As a consequence, the volume functional $\mathcal{V}$ is also concave on $\bar{P}$ and Theorem 1.1 holds if the maximum of $\mathcal{V}$ is interior. Next we explore the behavior of $\mathcal{V}$ near the boundary of $\bar{P}$.

Proposition 6.7 (Simple degeneracy) If $\left(Q_{t}\right)_{t \geq 0}$ is a smooth family of ideal tetrahedra with dihedral angles $x_{t}, y_{t}, z_{t}$ such that $x_{0}, y_{0} \in(0, \pi) ; z_{0}=0$ and $\left.\frac{d z_{t}}{d t}\right|_{t=0}>0$, then $\left.\frac{d \mathcal{V}}{d t}\right|_{t=0}=+\infty$.

Proof Simply check that the right hand side of (5) goes to 0 as $t$ goes to 0 . We call this situation simple degeneracy because the limiting triangle has only one vanishing angle (two of its vertices are therefore collapsed).

Proposition 6.8 (Double degeneracy) If $\left(Q_{t}\right)_{t \geq 0}$ is a smooth family of ideal tetrahedra satisfying $\left(x_{0}, y_{0}, z_{0}\right)=(0,0, \pi)$ and $\left.\frac{d}{d t}\right|_{t=0}\left(x_{t}, y_{t}, z_{t}\right)=(1+\lambda, 1-\lambda,-2)$ with $\lambda \in(-1,1)$, then

$$
\left.\exp \frac{-d \mathcal{V}}{d t}\right|_{t=0}=\frac{1-\lambda^{2}}{4}\left(\frac{1+\lambda}{1-\lambda}\right)^{\lambda}
$$


Proof As $t$ goes to 0 , one has $\sin x_{t} \sim(1+\lambda) t$ and $\sin y_{t} \sim(1-\lambda) t$ and $\sin z_{t} \sim 2 t$. The right hand side of (5) is thus equivalent to

$$
((1+\lambda) t)^{1+\lambda}((1-\lambda) t)^{1-\lambda}(2 t)^{-2}=\frac{1-\lambda^{2}}{4}\left(\frac{1+\lambda}{1-\lambda}\right)^{\lambda}
$$

We call this situation double degeneracy because the limiting triangle has two vanishing angles (its vertices are distinct, but collinear). At a double degeneracy, the volume has directional derivatives, but no well-defined differential.

\section{Ruling out some degeneracies}

From now on, we fix $\left(w_{i}\right)$ in the compact polyhedron $\bar{P}$ at a point which maximizes the total hyperbolic volume of all tetrahedra. To prove that $\left(w_{i}\right)$ is critical for the volume $\mathcal{V}$, we only need to make sure that $\left(w_{i}\right)$ lies in the interior $P$ of $\bar{P}$, ie that all $x_{i}, y_{i}, z_{i}$ lie in $(0, \pi)$.

Proposition 7.1 If for some index $i$, one of the numbers $x_{i}, y_{i}, z_{i}$ is 0 , then two of them are 0 and the third is $\pi$. In other words, there are no simple degeneracies, only double degeneracies.

Proof If not, consider an affine segment from $\left(w_{i}\right)$ to some interior point of $P$. By Proposition 6.7, the partial derivative of $\mathcal{V}$ at $\left(w_{i}\right)$ along that segment is not bounded above, so $\mathcal{V}$ was not maximal at $\left(w_{i}\right)$.

Tetrahedra $\Delta_{i}$ such that $\left(x_{i}, y_{i}, z_{i}\right)$ has one, and therefore two vanishing terms are called flat, and are characterized by the property that $w_{i}$ is either 0 or $\frac{\pi}{2}$.

Proposition 7.2 (Domino effect) If two consecutive tetrahedra $\Delta_{i-1}, \Delta_{i}$ are flat, then $\Delta_{i+1}$ is flat, too.

Proof We use only Table 1 and the deductions recorded in (3). There are three cases:

(1) If $i$ is not a hinge index, flatness of $\Delta_{i}$ implies $w_{i-1}+w_{i+1} \in\{0, \pi\}$. By the range condition $0 \leq w \leq \frac{\pi}{2}$, this implies $w_{i+1} \in\left\{0, \frac{\pi}{2}\right\}$, so $\Delta_{i+1}$ is flat.

(2) If $i$ is a hinge index and $w_{i}=\frac{\pi}{2}$, we must have $\left|w_{i-1}-w_{i+1}\right|=\frac{\pi}{2}$, so by the range condition, $w_{i+1}$ is 0 or $\frac{\pi}{2}$, and $\Delta_{i+1}$ is flat.

(3) If $i$ is a hinge index and $w_{i}=0$, we have $\left|w_{i+1}-w_{i-1}\right| \leq 0$ so $w_{i+1}=w_{i-1}$. But $\Delta_{i-1}$ is assumed flat, and therefore so is $\Delta_{i+1}$. Note that flatness of $\Delta_{i-1}$ was needed only in this case. 
Proposition 7.3 If $\Delta_{i}$ is flat, then $i$ is a hinge index and $w_{i}=0$.

Proof In all other cases, the proof of Proposition 7.2 actually forces $\Delta_{i+1}$ to be flat, which triggers a domino effect: all $\Delta_{j}$ 's are flat, and the volume is $0-$ certainly not maximal.

Easy Fact 7.4 If $A B C$ is a Euclidean triangle with positive angles and edge lengths $a, b, c$, then

$$
\begin{aligned}
& a=b \Longleftrightarrow \widehat{A}=\widehat{B} \Longleftrightarrow \sin \widehat{A}=\sin \widehat{B} \\
& a<b \Longleftrightarrow \widehat{A}<\widehat{B} \Longleftrightarrow \sin \widehat{A}<\sin \widehat{B} .
\end{aligned}
$$

The volume $\mathcal{V}$ is still supposed maximal, and we assume that some tetrahedra $\Delta_{i}$ are flat, ie that some hinge parameters $w_{i}$ vanish. Places where a parameter $w_{i}$ vanishes will be signalled by a vertical bar: ... $L L \mid R R \ldots$ By Proposition 7.2, consecutive vertical bars are always separated by at least two letters.

The patterns $R L \mid R L$ and $L R \mid L R$ can never occur, because increasing the incriminated $w_{i}$ to $\varepsilon$ would automatically increase the volume (note that $w_{i-1}=w_{i+1}=: A$ by the hinge condition (3)-iii):

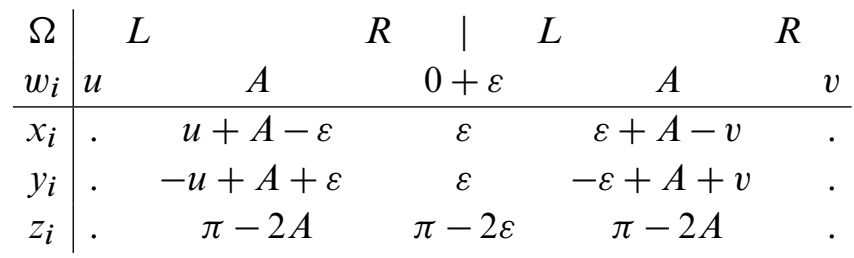

This implies

$$
\left.\exp \frac{-\partial \mathcal{V}}{\partial \varepsilon}\right|_{\varepsilon=0}=\frac{1}{4} \cdot \frac{\sin (A-u)}{\sin (A+u)} \frac{\sin (A-v)}{\sin (A+v)}<1
$$

where we used Easy Fact 7.4, Proposition 6.1 and Proposition 6.8 (with $\lambda=0$ ).

Any vertical bar thus lives next to at least two consecutive identical letters (on at least one side). However, the patterns $R|L L| R$ and $L|R R| L$ are also prohibited by Proposition 7.3, since the central (nonhinge) tetrahedron would have one vanishing angle $(a+c=0$ in the notations of Table 1$)$.

\section{A geometrical lemma}

Definition 8.1 In the universal cover of the tessellation $\mathcal{A}$ of the torus at infinity of $V_{\varphi}$ (Figure 4), a fan is a sequence of at least three consecutive layers, such that the first and last layers are grey and all layers in between are white. Fans are in bijection 
with the subwords of $\Omega$ of the form $R L^{k} R$ or $L R^{k} L$ with $k \geq 2$ (see the remarks to Figure 4 in Section 4).

Lemma 8.2 Suppose $w_{0}=0$, so that $\Omega$ contains a subword $L \mid R^{k} L$ with $k \geq 2$, or $L\left|R^{k}\right| L$ with $k \geq 3$ (in the latter case, the second bar indicates that $w_{k}$ vanishes as well as $\left.w_{0}\right)$. The corresponding fan admits a complete Euclidean structure with boundary (with angles prescribed by the $w_{i}$ 's). Moreover, let $Q, P, T$ be the lengths of the segments of the broken line corresponding to the first $R$, in the order indicated in Figure $9(P, T$ are the sides adjacent to the apex in a flat upward-pointing grey [hinge] triangle, in the sense of Figure 3). Then $Q<P+T$.

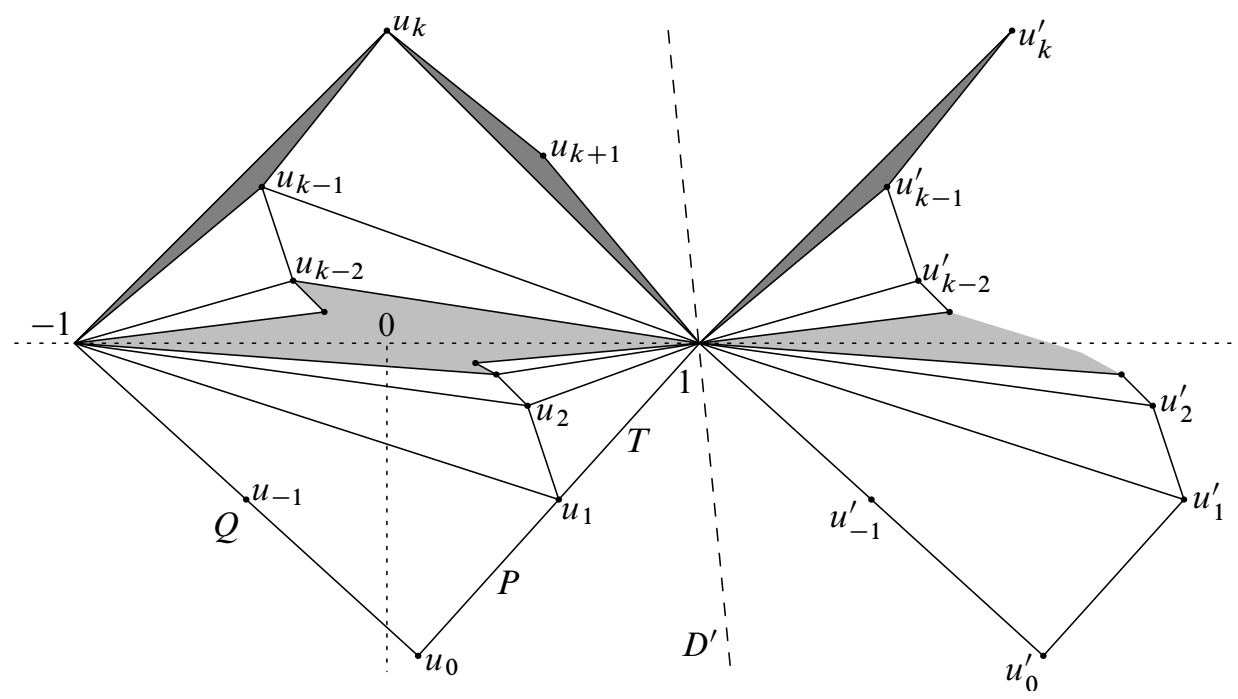

Figure 9: The situation where $Q \geq P+T$ cannot hold

Proof We first restrict our attention to the case $L \mid R^{k} L, k \geq 2$. The interior vertices of the topological fan correspond to the indices $i$ living between two $R$ 's, ie $1 \leq i \leq k-1$, in the sense that holonomy around the $i$-th vertex $u_{i}$ (Figure 9) is controlled by $\partial \mathcal{V} / \partial w_{i}$ (Sublemma 6.4). When $2 \leq i \leq k-1$, none of the triangles adjacent to $u_{i}$ are flat, so $w_{i}$ can vary in a small interval without making the $m$-tuple $w$ exit the domain $\bar{P}$; consequently, the value of $w_{i}$ in that interval is critical, which by the first case (nonhinge) of Sublemma 6.4 implies that the holonomy around the associated vertex $u_{i}$ is trivial. As for $i=1$, the corresponding vertex $u_{1}$ is adjacent to a flat angle $z_{0}=\pi$ (Figure 9) so its holonomy is not imposed by the $w_{i}$ 's (when a triangle has an angle $\pi$, the other two angles are always 0 while the adjacent sides may have arbitrary lengths). 
The case of $L\left|R^{k}\right| L$ is similar: for indices $2 \leq i \leq k-2$, Sublemma 6.4 applies, while for $i=1$ or $k-1$ holonomy is not imposed by the $w_{i}$ 's.

Therefore we can embed the fan as an infinite necklace of triangulated polygons into $\mathbb{C}$. We shall no longer distinguish $L \mid R^{k} L$ from $L\left|R^{k}\right| L$ here, and shall formulate all properties in terms of complex numbers in order to keep track of oriented angles. We make two consecutive nodes (ie lifts of the $(2 k+4)$-valent vertex of $\mathcal{A}$ associated to the full subword $L R^{k} L$ ) coincide with -1 and 1 in $\mathbb{C}$, and also denote by $u_{i}$ the complex coordinate of the copy of $u_{i}$ between these nodes (the index $i$ actually ranges from -1 to $k+1$; see Figure 9). Incidentally, one can show that the $u_{i}$ form (part of) an orbit of a certain deck transformation of the universal cover $\mathbb{H}^{3} \rightarrow V_{\varphi}$. We will discuss this more at the end of Section 10.

We arrange matters so that $\operatorname{Im}\left(u_{0}\right)<0$ and $u_{1}$ lies on the open segment $\left(u_{0}, 1\right)$. While removing any node disconnects the fan, Condition (1)-iii implies that the image of the embedding is invariant under a horizontal translation of length 2 . In particular, the geometric link of each node, such as 1 or -1 , is completely determined. To prove the assertion of the Lemma, it is sufficient to show that $\operatorname{Re}\left(u_{0}\right)<0$. Assume $\operatorname{Re}\left(u_{0}\right) \geq 0$ (so $u_{0}$ lies in the lower-right quadrant) and aim for a contradiction.

The similarity property of the triangles with vertices $1, u_{i}, u_{i+1}$ and $-1, u_{i}, u_{i-1}$ is expressed by the relation $\frac{u_{i+1}-1}{u_{i}-1}=\frac{u_{i-1}+1}{u_{i}+1}$, hence by induction

$$
\left(u_{i+1}-1\right)\left(u_{i}+1\right)=\left(u_{i}-1\right)\left(u_{i-1}+1\right)=\cdots=\left(u_{1}-1\right)\left(u_{0}+1\right)=: K .
$$

Then, as $u_{1}$ sits between 1 and $u_{0}$, the number $u_{1}-1$ is a positive (real) multiple of $u_{0}-1$, so $K$ is a positive multiple of $u_{0}^{2}-1$ which implies $\operatorname{Im}(K) \leq 0$. Let $D$ be the line through 0 and the points $\pm \sqrt{K}$ : either $D$ is vertical, or $D$ visits the upper-left and lower-right (open) quadrants. Let $D^{\prime}$ be the line through 1 , parallel to $D$; and define $u_{i}^{\prime}:=2+u_{i}$ for all $i$. By definition of $K$, the rays $\left[1, u_{i+1}\right)$ and $\left[1, u_{i}^{\prime}\right)$ are symmetric with respect to $D^{\prime}$. Moreover, the rays through $u_{0}, u_{1}, \ldots, u_{k}, u_{k+1}, u_{k}^{\prime}, u_{k-1}^{\prime}, \ldots, u_{0}^{\prime}, u_{-1}^{\prime}, u_{0}$ issuing from 1 (in that cyclic order) divide $\mathbb{C}$ into angular sectors of sum $2 \pi$ realizing the geometric link of a node of the fan, as specified by the angles $x_{i}, y_{i}, z_{i} \geq 0$. Finally, since all these angles are nonnegative, the symmetry of the link with respect to $D^{\prime}$ implies that for all $-1 \leq i \leq k$, the point $u_{i}^{\prime}$ (resp. $u_{i+1}$ ) is on the right (resp. left) of $D^{\prime}$.

Recall $\operatorname{Im}\left(u_{0}\right)<0$. Suppose by induction $\operatorname{Im}\left(u_{i}\right)<0$ for some $0 \leq i \leq k$. Then $\operatorname{Im}\left(u_{i}^{\prime}\right)<0$. Considering the direction of $D^{\prime}$ and the symmetry property with respect to $D^{\prime}$, this implies $\operatorname{Im}\left(u_{i+1}\right)<0$. By an immediate induction, the angular sector $\widehat{u_{k+1} 1 u_{k}^{\prime}}$ (just above 1 ) is larger than $\pi$. But it is an angle of the link at the node 1 (namely, $z_{k+1}$ ), giving a contradiction. 
Of course, a statement similar to Lemma 8.2 holds for subwords $L R^{k} \mid L$, and also for $R\left|L^{k} R, R\right| L^{k} \mid R$ and $R L^{k} \mid R$.

\section{Ruling out all degeneracies}

Easy Fact 9.1 If $U$ and $V$ are positive constants, the function defined on $(-1,1)$ by

$$
f(\lambda):=\frac{1-\lambda^{2}}{4} U\left(\frac{1+\lambda}{1-\lambda} V\right)^{\lambda}
$$

takes the value $\frac{U}{(1+V)\left(1+V^{-1}\right)}$ for some $\lambda$. It is in fact an absolute minimum: indeed,

$$
(\log f)^{\prime}(\lambda)=\log \left[\frac{1+\lambda}{1-\lambda} V\right],
$$

so $f$ is minimal when the bracket is 1 , and the result follows by direct computation.

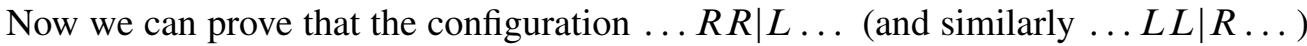
never occurs, which will imply Theorem 1.1. The strategy is to suppose $R R \mid L$ appears, ie $w_{j}=0$ for some $j$ (for notational convenience we assume $j=2$ ). Next, replace $w_{2}$ by $\varepsilon$ and $w_{1}$ by $w_{1}+\lambda \varepsilon$, for a wisely chosen $\lambda$. The volume $\mathcal{V}$ will then increase. (The value of $\lambda$, which does not need to be explicitly computed, will maximize $\partial \mathcal{V} / \partial \varepsilon$, and $e^{-\partial \mathcal{V} / \partial \varepsilon}$ will be the value of $f$ given in Easy Fact 9.1. We will specify in due time what the parameters $U, V$ are.) Volume computations follow from Proposition 6.8 (at the index $i=2$ ) and Proposition 6.1 (other indices).

\subsection{Case 1: $R R \mid L R$}

According to Table 1 , the angles are as follows. Note the relation $w_{1}=w_{3}=: A$, a

\begin{tabular}{|c|c|c|c|c|c|}
\hline$\Omega$ & & & | & & $R$ \\
\hline$i$ & 0 & 1 & 2 & 3 & 4 \\
\hline$w_{i}$ & $u$ & $A+\lambda \varepsilon$ & $0+\varepsilon$ & $A$ & 2 \\
\hline$x_{i}$ & $\xi-A-\lambda \varepsilon$ & $-u+2 A+2 \lambda \varepsilon-\varepsilon$ & $(1-\lambda) \varepsilon$ & $\varepsilon+A-v$ & \\
\hline$y_{i}$ & $\eta+A+\lambda \varepsilon$ & $u+\varepsilon$ & $(1+\lambda) \varepsilon$ & $-\varepsilon+A+v$ & \\
\hline$z_{i}$ & $\pi-2 u$ & $\pi-2 A-2 \lambda \varepsilon$ & $\pi-2 \varepsilon$ & $\pi-2 A$ & \\
\hline
\end{tabular}
consequence of the hinge condition (3)-iii.

We have $u=w_{0}>0$ because $L|R R| L R$ is impossible (according to the discussion after Easy Fact 7.4). Thus, $\Delta_{0}$ is not flat: if $-1<\lambda<1$, then $\varepsilon$ can take small positive 
values without making any of the $x_{i}, y_{i}, z_{i}$ negative. With the correct choice of $\lambda$, we deduce

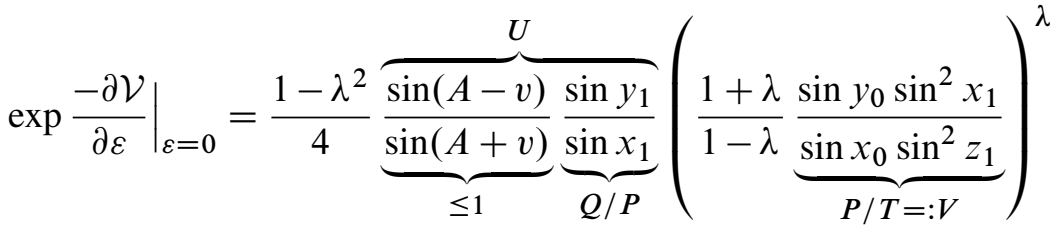

$$
\begin{aligned}
& \leq \frac{Q / P}{(1+P / T)(1+T / P)}=\frac{1}{1+P / T} \frac{Q}{P+T}<1
\end{aligned}
$$

by Lemma 8.2 (see Figure 10 (left) - again, the sine relation in triangles was used to compute $Q / P$ and $P / T)$.

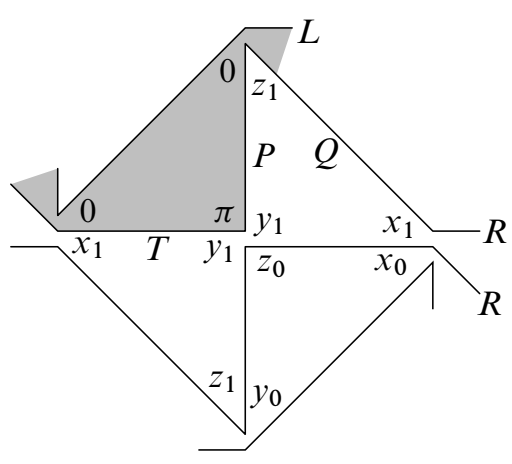

Case 1

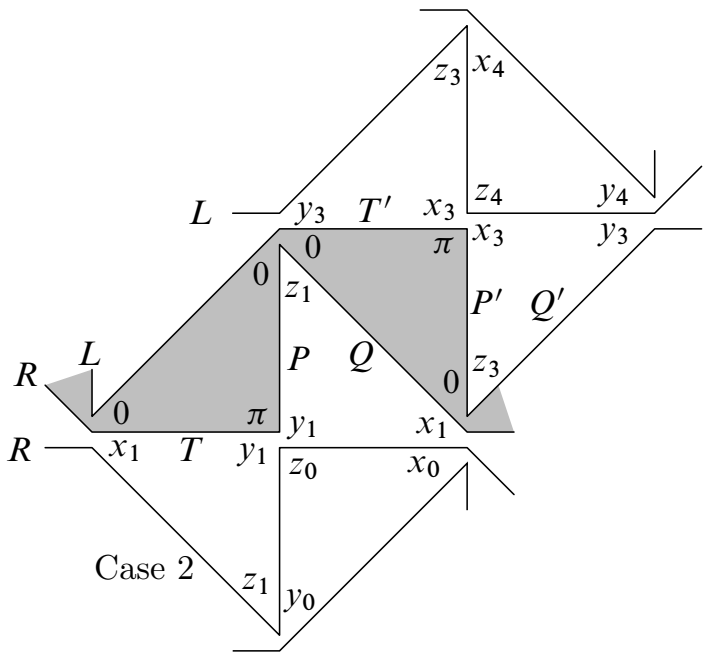

Figure 10

\subsection{Case 2: $R R \mid L L$}

\begin{tabular}{c|cccccc}
$\Omega$ & & $R$ & & $R$ & $\mid$ & $L$ \\
& $L$ \\
$i$ & 0 & 1 & 2 & 3 & 4 \\
$w_{i}$ & $u$ & $A+\lambda \varepsilon$ & $0+\varepsilon$ & $A$ & $v$ \\
\hline$x_{i}$ & $\xi-A-\lambda \varepsilon$ & $-u+2 A+2 \lambda \varepsilon-\varepsilon$ & $(1-\lambda) \varepsilon$ & $\varepsilon+v$ & $A+\xi^{\prime}$ \\
$y_{i}$ & $\eta+A+\lambda \varepsilon$ & $u+\varepsilon$ & $(1+\lambda) \varepsilon$ & $-\varepsilon+2 A-v$ & $-A+\eta^{\prime}$ \\
$z_{i}$ & $\pi-2 u$ & $\pi-2 A-2 \lambda \varepsilon$ & $\pi-2 \varepsilon$ & $\pi-2 A$ & $\pi-2 v$
\end{tabular}

First consider the value of $A$. Since there can be no vertical bars immediately before or after $R R \mid L L$, the tetrahedra $\Delta_{0}, \Delta_{1}, \Delta_{3}, \Delta_{4}$ have positive angles, so the parameter 
$A$ (which does not contribute to the angles of any other tetrahedra) can vary freely in an open interval when $\varepsilon=0$. So $A$ must be critical giving

$$
1=\left.\exp \frac{-\partial \mathcal{V}}{\partial A}\right|_{\varepsilon=0}=\underbrace{\frac{\sin y_{0} \sin ^{2} x_{1}}{\sin x_{0} \sin ^{2} z_{1}}}_{P / T} \underbrace{\frac{\sin ^{2} y_{3} \sin x_{4}}{\sin ^{2} z_{3} \sin y_{4}}}_{P^{\prime} / T^{\prime}} .
$$

Hence $P / T=T^{\prime} / P^{\prime}$. Therefore, with the right choice of $\lambda$,

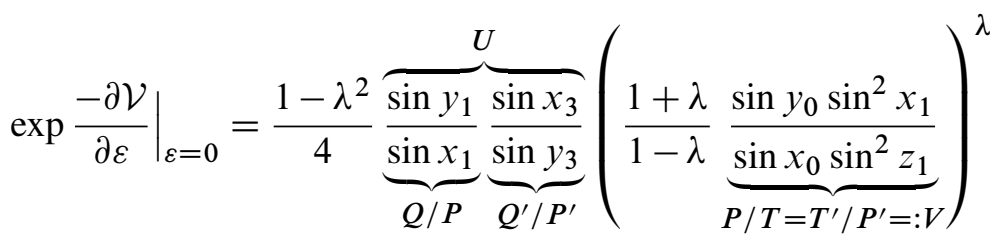

$$
\begin{aligned}
& =\frac{Q / P \cdot Q^{\prime} / P^{\prime}}{(1+T / P)\left(1+T^{\prime} / P^{\prime}\right)}=\frac{Q}{P+T} \frac{Q^{\prime}}{P^{\prime}+T^{\prime}}<1
\end{aligned}
$$

by Lemma 8.2; see Figure 10 (right).

We conclude with two remarks. First, up to replacing the monodromy $\varphi$ with $\varphi^{2}$ (thus doubling the period $m$ of $\Omega$ ), we can always assume $\Omega$ has at least 6 letters: that way, all columns of the tables above are neatly distinct, and to recover the original $V_{\varphi}$ we just quotient out by the extra symmetry (which the volume maximizer $\left(w_{i}\right)_{i \in \mathbb{Z} / m \mathbb{Z}}$ must respect, by concavity of the volume functional $\mathcal{V})$. Compare with the remark that closes Section 5. Second, the choice of $\lambda \in(-1,1)$, which may seem "magical" at first glance, is essentially our only degree of freedom in Section 9.1-Section 9.2: the volume is already assumed critical with respect to most parameters (including $A$, the common value of $w_{1}$ and $w_{3}$ ); therefore, only deformations of $w_{1}, w_{2}, w_{3}$ need to be considered, and if we assume $\frac{\partial w_{2}}{\partial \varepsilon}=1$, then only the value of the difference $\frac{\partial w_{1}}{\partial \varepsilon}-\frac{\partial w_{3}}{\partial \varepsilon} \in(-1,1)$ matters.

Theorem 1.1 is proved.

\section{A numerical example: $R^{N} L^{M}$}

In this section we fix two large enough integers $N$ and $M$ and investigate the behavior of the angles for $\Omega=R^{N} L^{M}$ : the angles made positive by the previous computations will turn out to be very small. We will directly construct a Euclidean realization of Figure 4, automatically unique up to isometry. Since $N$ and $M$ are large, there exist small complex numbers $a, a^{\prime}, b, b^{\prime}$ such that

$$
\left\{\begin{array} { l } 
{ \operatorname { s i n } a = i \operatorname { t a n } b \operatorname { c o s } b ^ { \prime } } \\
{ \operatorname { s i n } a ^ { \prime } = - i \operatorname { t a n } b ^ { \prime } \operatorname { c o s } b }
\end{array} \text { where } \quad \left\{\begin{array}{l}
b=(\pi-2 a) / N \\
b^{\prime}=\left(\pi-2 a^{\prime}\right) / M .
\end{array}\right.\right.
$$


A way to compute $a, a^{\prime}$ is to set $a_{0}=a_{0}^{\prime}=0$ and to define inductively

$$
a_{s+1}=\arcsin \left(i \tan \frac{\pi-2 a_{s}}{N} \cos \frac{\pi-2 a_{s}^{\prime}}{M}\right)
$$

and a similar expression for $a_{s+1}^{\prime}$, with a change of sign. The sequences $a_{s}, a_{s}^{\prime}$ converge exponentially fast to $a, a^{\prime}$. The constants $a, a^{\prime}$ become arbitrarily small for large enough $N, M$, hence $b \sim \pi / N$ and $b^{\prime} \sim \pi / M$. So plugging into (6), $a \sim i \pi / N$ and $a^{\prime} \sim-i \pi / M$. Using the Landau symbol $O(A, B)$ in the sense of $O(\max \{A, B\})$, this in turn yields

$$
\begin{array}{rlrl}
b & =\frac{\pi}{N}-\frac{2 i \pi}{N^{2}}+O\left(\frac{1}{N^{3}}, \frac{1}{M^{3}}\right) & a & =\frac{i \pi}{N}+\frac{2 \pi}{N^{2}}+O\left(\frac{1}{N^{3}}, \frac{1}{M^{3}}\right) \\
b^{\prime} & =\frac{\pi}{M}+\frac{2 i \pi}{M^{2}}+O\left(\frac{1}{N^{3}}, \frac{1}{M^{3}}\right) & a^{\prime}=\frac{-i \pi}{M}+\frac{2 \pi}{M^{2}}+O\left(\frac{1}{N^{3}}, \frac{1}{M^{3}}\right) .
\end{array}
$$

(In fact $a, a^{\prime}, b, b^{\prime}$ are analytic functions of $\frac{1}{N}, \frac{1}{M}$, by the Implicit Function Theorem.)

Proposition 10.1 The fan which corresponds to $R^{N}$ can be embedded into $\mathbb{C}$ with nodes at complex coordinates $\pm \cot b$ and intermediary vertices $\cot (a+s b)$ where $-1 \leq s \leq N+1$; similarly, the fan corresponding to $L^{M}$ can be embedded into $\mathbb{C}$ (possibly with a different scaling factor) with nodes $\pm \cot b^{\prime}$ and intermediary vertices $\cot \left(a^{\prime}+s b^{\prime}\right)$ where $-1 \leq s \leq M+1$ (see Figure 11).
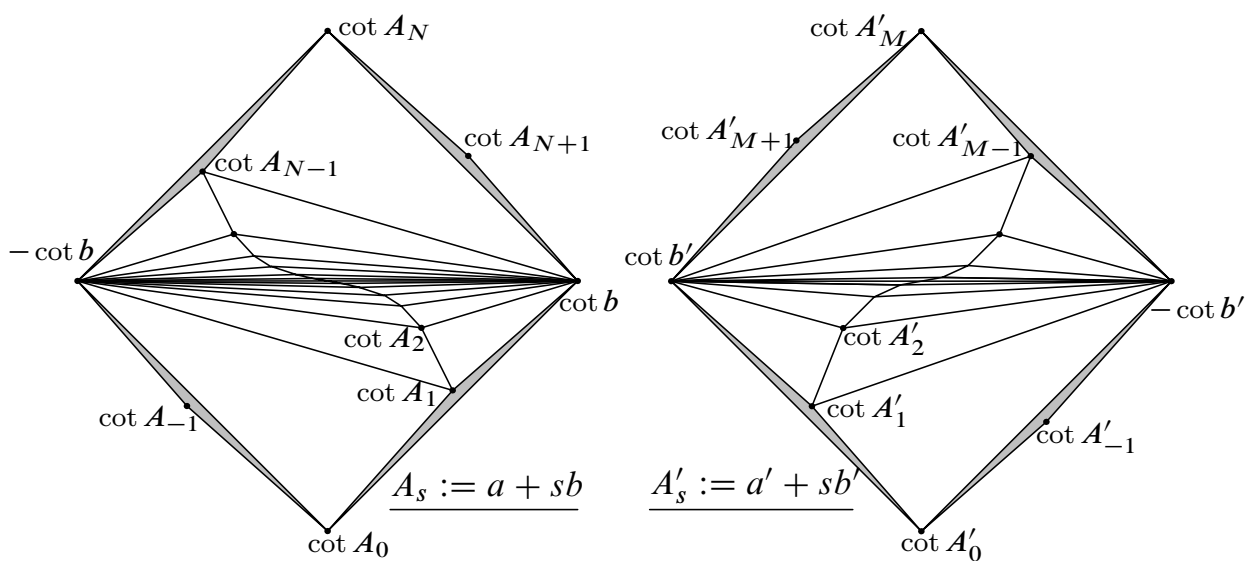

Figure 11 
Proof There are several things to check. First, the congruence of pairs of triangles inside each fan follows from the identity of complex ratios

$$
\frac{\cot \left(A_{s}+b\right)-\cot b}{\cot \left(A_{s}+b\right)-\cot A_{s}}=\frac{\cot \left(A_{s}-b\right)+\cot b}{\cot \left(A_{s}-b\right)-\cot A_{s}}=\frac{\sin ^{2} A_{s}}{\sin ^{2} b}
$$

where $A_{s}=a+s b$ for $0 \leq s \leq N$, and an identical relation for $a^{\prime}, b^{\prime}$.

Next, each fan, when stripped of two of its four limiting (grey) triangles, is a parallelogram. This follows from $\cot (a+N b)=\cot (\pi-a)=-\cot a$, and the same for $a^{\prime}$. In particular, each fan admits a center of symmetry.

Furthermore, these two parallelograms are congruent. To see this, let $\alpha, \alpha^{\prime}, \beta, \beta^{\prime}$ denote the squared cotangents of $a, a^{\prime}, b, b^{\prime}$. Raising (6) to the power -2 , we get

$$
\left\{\begin{array}{l}
1+\alpha=-\beta\left(1+\beta^{\prime-1}\right) \\
1+\alpha^{\prime}=-\beta^{\prime}\left(1+\beta^{-1}\right)
\end{array} \quad \text { hence } \quad \frac{\alpha}{\alpha^{\prime}}=\frac{\beta+\beta / \beta^{\prime}+1}{\beta^{\prime}+\beta^{\prime} / \beta+1}=\frac{\beta}{\beta^{\prime}},\right.
$$

so $\cot a / \cot b= \pm \cot a^{\prime} / \cot b^{\prime}$, the correct sign being minus by the estimates (7).

Further yet, the limiting (grey) triangles of the two fans have the same shape: by (8) their complex ratios are $\sin ^{2} a / \sin ^{2} b$ and $\sin ^{2} b^{\prime} / \sin ^{2} a^{\prime}$, both equal by (6) to $-\cos ^{2} b^{\prime} / \cos ^{2} b$.

Finally, all triangles are positively oriented, that is, $\operatorname{Im}\left(\sin ^{2} A_{s} / \sin ^{2} b\right)>0$ for all $0 \leq s \leq N$. We first check this for $s=0$ (the case $s=N$ will follow by symmetry): we have $\sin ^{2} a / \sin ^{2} b=-\left(\cos ^{2} b^{\prime} / \cos ^{2} b\right)=-\left(\left(1-\sin ^{2} b^{\prime}\right) /\left(1-\sin ^{2} b\right)\right)$. In the latter expression, both the numerator and denominator are $\sim 1$, but their imaginary parts are equivalent to $-4 \pi^{2} / M^{3}$ and $4 \pi^{2} / N^{3}$ respectively (with an $O\left(N^{-4}, M^{-4}\right.$ ) error), by (7). Therefore, the ratio does lie above the real line, and the "pinched" angles of the limiting (grey) triangles in Figure 11 are both roughly

$$
2 \pi^{2}\left(N^{-3}+M^{-3}\right) \text { radians. }
$$

Very pinched, but not flat!

To check that $\arg \left(\sin A_{s} / \sin b\right)$ lies in $(0, \pi / 2)$ for all other $0 \leq s \leq N$, we need to draw the level curves of $z \mapsto \arg (\sin z)$ in $\mathbb{C}$. This is done in Figure 12, in the case $0 \leq \operatorname{Re}(z) \leq \pi$ : the curves fall into 4 symmetric families ("quadrants" meeting at $\pi / 2 \in \mathbb{C}$ ), and it is an easy exercise to check that the families above (resp. below) the real axis are made of convex (resp. concave) curves. The authorized region for the $A_{s}=a+s b$ is in grey (with a narrow collar near $\pi / 2$ ); the forbidden regions are left in white. The segment $\left[A_{0} A_{N}\right]$ clearly stays in the grey region, which implies the result. 


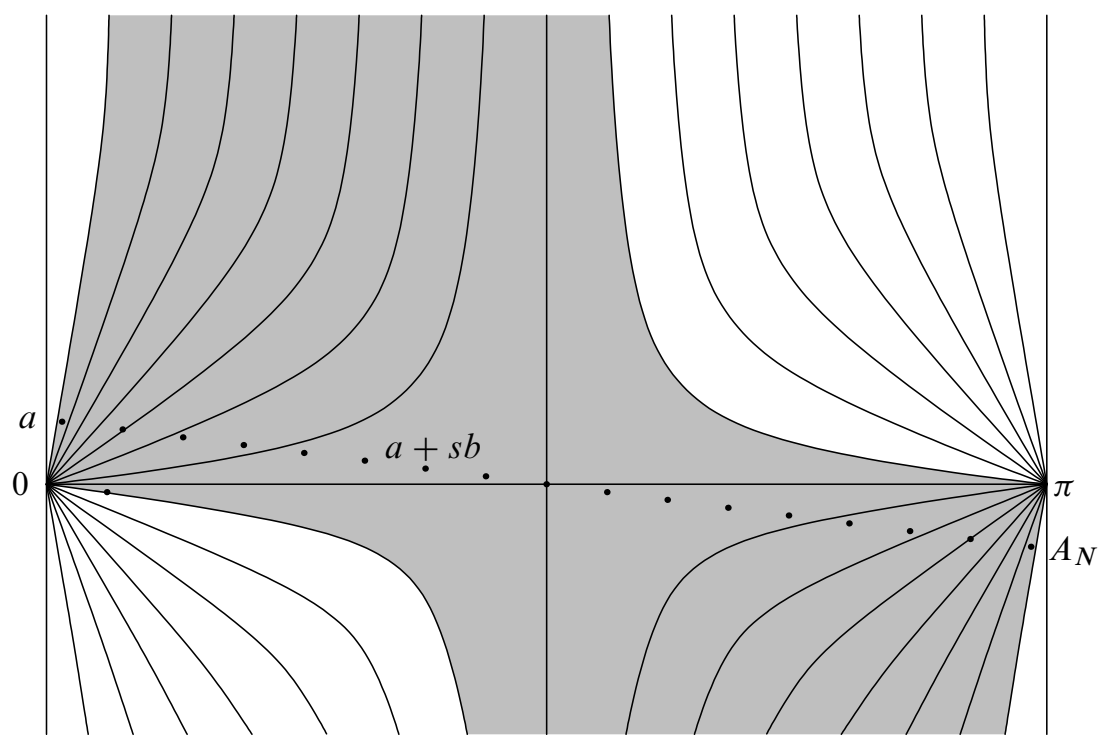

Figure 12: Level curves of $z \mapsto \arg (\sin z)$

By the same argument, the triangles in the fan of $L^{M}$ are well-oriented, too. Therefore we may tile the plane with parallelograms (or fans) congruent to those in Figure 11 to get a Euclidean realization of Figure 4.

Finally, notice that the Kleinian group associated to the embedding of the left fan in Figure 11 contains the Möbius transformation $z \mapsto(z \cos b-\sin b /(z \sin b+\cos b))$ (it sends each tetrahedron sitting above a triangle in the left half of the fan to the tetrahedron sitting above the similar triangle in the right half). Therefore, $2 i b$ (and similarly $-2 i b^{\prime}$ ) are the complex lengths of very short closed geodesics in the hyperbolic manifold $V_{\varphi}$.

A consequence of Theorem 1.1 is that the volume of any angle structure (defined by Table 1, where (3) holds) is a lower bound for the volume of the manifold $V_{\varphi}$. One gets bounds which are sharp in terms of distances in the Farey graph; see Section B.1.

\section{Once-punctured tori and 4-punctured spheres}

Theorem 1.1 is still true if we replace the once-punctured torus $T$ by the 4 -punctured sphere $S$, and the map $\varphi: T \rightarrow T$ by an orientation-preserving homeomorphism $\varphi_{S}: S \rightarrow S$ (of course, we must specify how the "eigenvalues" of $\varphi_{S}$ are defined). In fact, the tetrahedra of the resulting manifold $V_{\varphi_{S}}$ and of $V_{\varphi}$ are metrically the same; only the combinatorics of their gluing changes a little. 
Define $R:=\mathbb{R}^{2} \backslash \mathbb{Z}^{2}$ and the maps $\alpha, \beta, \sigma: R \rightarrow R$ characterized by $\alpha(x)=x+(1,0)$; $\beta(x)=x+(0,1) ; \sigma(x)=-x$. Then we have natural identifications $T=R /\langle\alpha, \beta\rangle$ and $S=R /\left\langle\alpha^{2}, \beta^{2}, \sigma\right\rangle$. Define also $T^{\prime}:=R /\left\langle\alpha^{2}, \beta^{2}\right\rangle$ (note that $T^{\prime}$ is a 4-punctured torus).

One can show that up to isotopy, any orientation-preserving diffeomorphism $\varphi_{S}$ of $S$ lifts to a map $\varphi_{R}: R \rightarrow R$ such that $\varphi_{R}(x)=M x+v$ for some $M \in \operatorname{SL}_{2}(\mathbb{Z})$ and $v \in \mathbb{Z}^{2}$. Moreover, $( \pm M)$ and $\left(v \bmod 2 \mathbb{Z}^{2}\right)$ are unique. So we may define the eigenvalues of $\varphi_{S}$ (up to sign) as those of $M$. Observe finally that $\varphi_{R}$ induces orientation-preserving diffeomorphisms $\varphi^{\prime}: T^{\prime} \rightarrow T^{\prime}$ and $\varphi_{T}: T \rightarrow T$.

There are obvious coverings $T^{\prime} \rightarrow T$ and $T^{\prime} \rightarrow S$, of degrees 4 and 2 respectively. Given an ideal triangulation $\tau_{T}$ of $T$ (corresponding to a Farey triangle), we can lift $\tau_{T}$ to an ideal triangulation $\tau^{\prime}$ of $T^{\prime}$. Observe that $\sigma$ acts on $T^{\prime}$ as a properly discontinuous involution fixing $\tau^{\prime}$, hence $\tau^{\prime}$ descends to a triangulation $\tau_{S}$ of $S$. It is easy to see that $\tau_{S}$ has the combinatorics of a tetrahedron. If $\tau_{T}$ and $\tau_{T}^{1}$ are separated by a diagonal exchange (see Section 3.1 for a definition), then $\tau_{S}$ and the corresponding $\tau_{S}^{1}$ are separated by two diagonal exchanges on opposite edges. Mutatis mutandis, the construction of Section 3 provides ideal (topological) triangulations of $T^{\prime} \times \mathbb{R}$ and $S \times \mathbb{R}$, as well as of their quotients $V_{\varphi^{\prime}}$ and $V_{\varphi_{S}}$. There are coverings $V_{\varphi^{\prime}} \rightarrow V_{\varphi_{S}}$ and $V_{\varphi^{\prime}} \rightarrow V_{\varphi_{T}}$ (of degrees 2 and 4 respectively), and all these manifolds are hyperbolic when $\varphi_{S}$ has distinct real eigenvalues.

\section{Appendix A Geometric triangulations of two-bridge link complements}

\section{DAVID FUTER}

This appendix applies Guéritaud's techniques to find geometric triangulations for the hyperbolic two-bridge knot and link complements. These ideal triangulations are, in essence, the monodromy triangulations of 4-punctured sphere bundles, closed off in a slightly different way. They were constructed and studied in great detail by Sakuma and Weeks [23]. Akiyoshi, Sakuma, Wada and Yamashita have announced a proof that these triangulations are, in fact, geometrically canonical [5], which is a stronger statement than our result.

We will begin by reviewing two-bridge links and these ideal triangulations. We will then explain how the methods of the preceding paper give linear angle structures for these triangulations and prove that the volume function is maximized in the interior of the space of angle structures. Finally, we will prove two corollaries of this argument: a two-sided bound on the volume of the link complement and a result about arcs in the plane being hyperbolic geodesics. 


\section{A.1 Braids and two-bridge links}

Let $S$ be a 4 -punctured sphere, visualized concretely as a square pillowcase with the corners removed. A 4-string braid between two pillowcases, one interior and one exterior, defines a so-called product region $S \times I$. We will restrict our attention to alternating braids in which the top right strand is free of crossings. (See Figure 13 (a).)

(a)

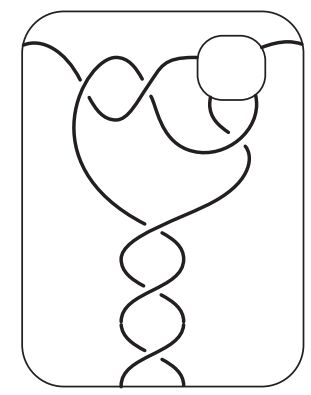

(b)

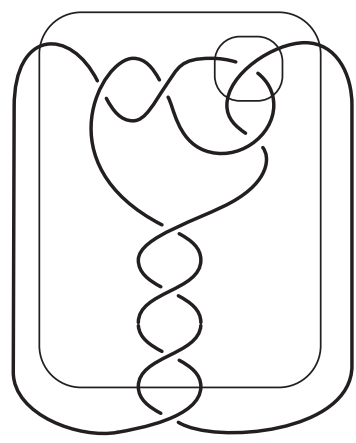

Figure 13: (a) An alternating braid between two pillowcases, described by the word $\Omega=R^{3} L^{2} R$ (b) The corresponding two-bridge link $K(\Omega)$

The mapping class $\varphi: S \rightarrow S$ induced by this braid can be described by a word

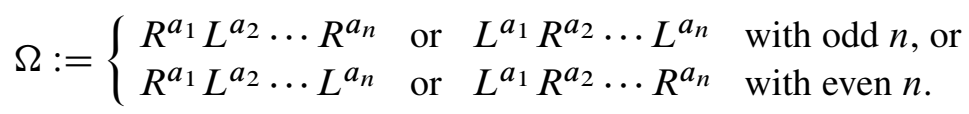

Here, $R$ encodes a crossing on the bottom pair of strands, and $L$ encodes a crossing on the left pair of strands, as in Figure 14. Each syllable of $\Omega$ (that is, each maximal subword $R^{a_{i}}$ or $L^{a_{i}}$ ) corresponds to a twist region in which two strands of the braid wrap around each other $a_{i}$ times. As we read $\Omega$ from left to right, we scan the crossings from the outside in. Note that, unlike the case of punctured torus bundles, our word $\Omega$ has a beginning and an end. For concreteness, we will focus on the case when $\Omega$ starts with $R$, as in Figure 13 (a); the $L$-case is similar.

An alternating braid of this sort can be completed to a link diagram, as follows. Outside the outer pillowcase, we connect the bottom left strand to the top right, and the bottom right strand to the top left, adding an extra crossing. (Up to isotopy of $\mathbb{S}^{2}$, there is a unique way to do this while keeping an alternating projection. In Figure 13 (b), the extra crossing was arbitrarily placed at the bottom of the diagram.) Similarly, inside the inner pillowcase we connect the strands in a diagonal fashion, adding an extra crossing while preserving the alternating projection. This creates an alternating link $K(\Omega)$, as in Figure 13 (b). 

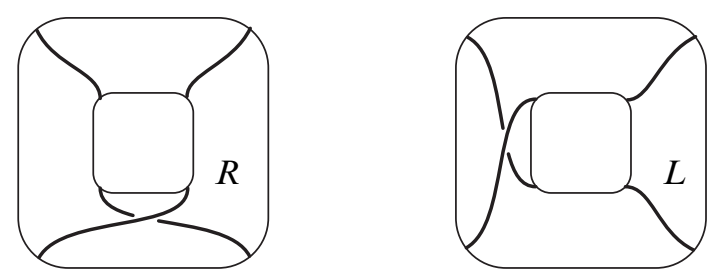

Figure 14: The letters $R$ and $L$ acting on strands of a braid

$K$ is called a two-bridge link because this diagram can be isotoped so that the pillowcases are horizontal, and the connecting strands form two bridges between the strands of the braid. It is well-known that, apart from the trivial link of one or two components, every two-bridge link can be constructed in this way. (See, for example, Murasugi [19, Theorems 9.3.1 and 9.3.2].)

William Menasco's theorem about hyperbolic alternating links [15] contains the following special case.

Theorem A.1 The two-bridge link $K(\Omega)$ is hyperbolic if and only if $\Omega$ has two or more syllables.

Just as with punctured torus bundles, we will give a direct proof of the "if" direction of this theorem by finding a geometric ideal triangulation of the link complement. The "only if" direction is immediate: a word with a single syllable produces a link with a single twist region, which must be a torus link.

\section{A.2 The ideal triangulation}

The word $\Omega$ describes a monodromy triangulation of the product region $S \times I$, in exactly the same fashion as for 4-punctured sphere bundles. In fact, because this product region is the complement of a braid in the part of $\mathbb{S}^{3}$ bounded by the two pillowcases, we can locate the edges of the triangulation concretely in the projection diagram.

Let $c=\sum_{i=1}^{n} a_{i}$ be the length of $\Omega$. Each letter $\Omega_{i}(1 \leq i \leq c)$, and thus each crossing in the alternating braid, corresponds to a 4 -punctured sphere $S_{i} \subset \mathbb{S}^{3} \backslash K$, with the four strands of $K$ seen in Figure 14 passing through the four punctures. The braid induces an ideal triangulation on each $S_{i}$, whose edges come from arcs in the diagram that look vertical and horizontal immediately before and/or after the corresponding crossing. See Figure 15. 

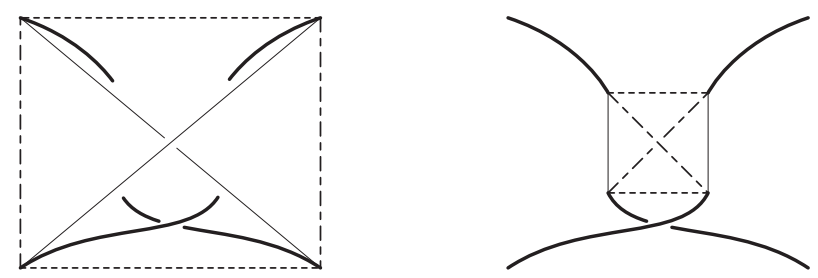

Figure 15: Two views of the same 4-punctured sphere $S_{i}$ living near a crossing in the link diagram (arcs with the same dashing pattern have the same slope)

Just as with punctured torus bundles, we can locate the progressively changing triangulations in the Farey tesselation $F$. Each triangulation of a 4-punctured sphere $S_{i}$, containing six edges of three different slopes, corresponds to a Farey triangle $t_{i}$. Triangles $t_{i}$ and $t_{i+1}$ share an edge $e_{i}$, whose endpoints are the shared slopes of $S_{i}$ and $S_{i+1}$. If $\Omega_{i}=R$, the path from $e_{i-1}$ to $e_{i}$ takes a right turn across $t_{i}$; if $\Omega_{i}=L$, the path takes a left turn. This rule also defines an initial edge $e_{0}$, because $\Omega_{1}=R$, so a right turn should take $e_{0}$ to $e_{1}$. Similarly, the action of $\Omega_{c}$ defines a terminal edge $e_{c}$.

For each $e_{i}, 1 \leq i \leq c-1$, the 4-punctured spheres $S_{i}$ and $S_{i+1}$ are joined together along four edges, two for each endpoint of $e_{i}$. In between them lies a layer $\Delta_{i}=\Delta\left(e_{i}\right)$ of two tetrahedra, whose bottom surface $S_{i}$ has the triangulation of $t_{i}$ and whose top surface $S_{i+1}$ has the triangulation of $t_{i+1}$. (See Figure 16 for an example.) Stacking these tetrahedron layers together produces a layered triangulation of the product region between $S_{1}$ and $S_{c}$.

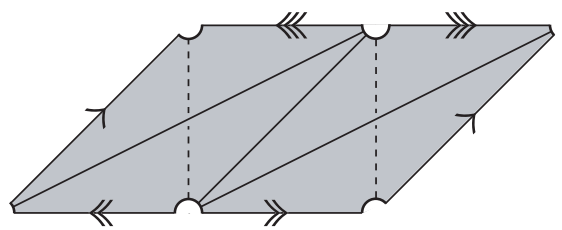

Figure 16: The tetrahedron layer $\Delta_{1}=\Delta\left(e_{1}\right)$, made of two tetrahedra contained between 4-punctured spheres $S_{1}$ and $S_{2}$ (sides with identical arrows are identified)

If we wanted to construct a bundle of 4-punctured spheres over the circle, we would glue the top of this product region to the bottom. To recover the complement of the link $K(\Omega)$, we follow a slightly different procedure. On the 4 -punctured sphere $S_{1}$, 
corresponding to the first crossing inside the outer pillowcase, let the peripheral edges be the edges whose slope is the vertex of $t_{1}$ opposite the initial edge $e_{0}$.

We will fold $S_{1}$ along the two peripheral edges, identifying its ideal triangles in pairs. Figure 17 shows that this creates exactly the desired effect of connecting the strands of $K$ in pairs, with a twist. This full twist corresponds to the first two crossings in the link projection: the first crossing in the braid, as well as the "extra" crossing outside the outer pillowcase. The four nonperipheral edges on $S_{1}$ are identified to a single edge, isotopic to a short arc near the crossing. The two ideal triangles that remain after folding are clasped together around this edge, which we call the core of the clasp.
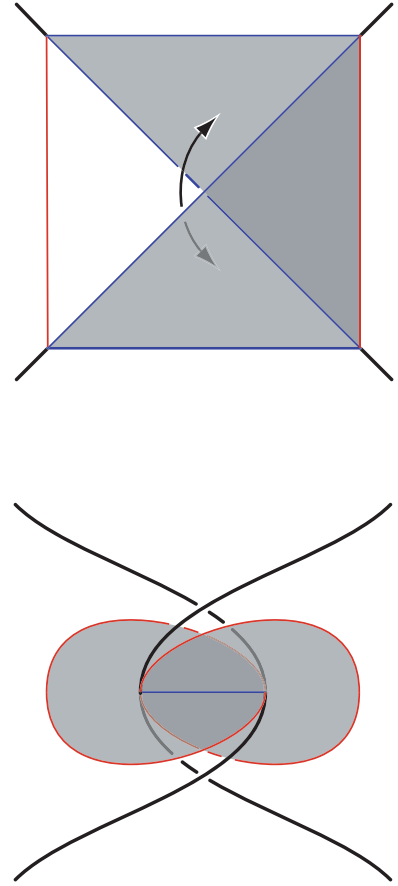

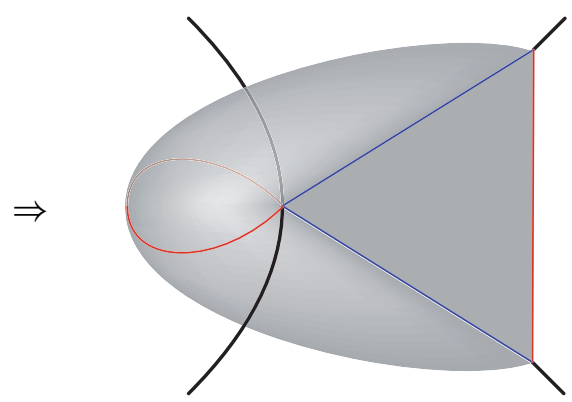

$\Downarrow \quad$ isotopy

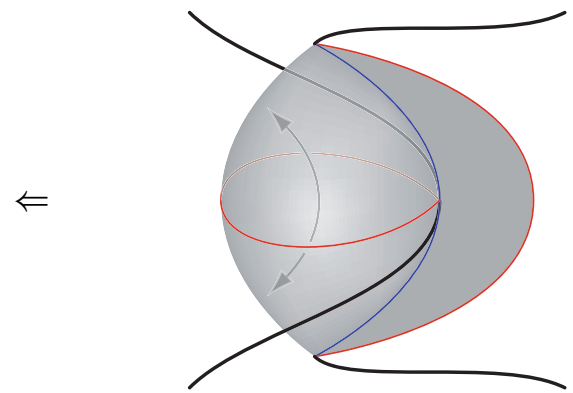

Figure 17: Folding the pleated surface $S_{1}$ produces the first two crossings in the link.

Let $\alpha$ be the mirror image of the peripheral slope across $e_{0}$ in the Farey graph. Topologically, folding $S_{1}$ as above amounts to attaching a thickened disk of boundary slope $\alpha$ to the outer pillowcase [23, Lemma II.2.5].

In a similar fashion, we define the peripheral edges on $S_{c}$ to be the edges whose slope is the vertex of $t_{c}$ opposite $e_{c}$. We fold $S_{c}$ along these two peripheral edges, identifying 
its faces to a clasp of two ideal triangles. Topologically, attaching 2-handles to $S_{1}$ and $S_{c}$ results in a space homeomorphic to the link complement. Combinatorially, folding $S_{1}$ and $S_{c}$ defines a gluing pattern for all the faces of the tetrahedra, giving us an ideal triangulation of $\mathbb{S}^{3} \backslash K$. See [23, Section II.2] for more details of this triangulation.

\section{A.3 Combinatorics at the cusp}

To describe the combinatorics of the boundary component(s) of $\mathbb{S}^{3} \backslash K$, we will first focus on the product region between the pleated surfaces $S_{1}$ and $S_{c}$. In the layered triangulation of this product region, each layer $\Delta_{i}$ consists of two tetrahedra, $D_{i}$ and $D_{i}^{\prime}$, as in Figure 16. It is clear from the figure that each tetrahedron has exactly one vertex at each puncture of $S_{i}$, ie at each strand of the 4-string braid between $S_{1}$ and $S_{c}$. Since the combinatorics of the four strands are identical, let us focus on a single puncture of the 4-punctured sphere.

The tetrahedron layer $\Delta_{i}$ intersects the neighborhood of a puncture in two boundary triangles, one from a truncated vertex of $D_{i}$ and one from $D_{i}^{\prime}$. These boundary triangles meet at two vertices that come from shared edges of $D_{i}$ and $D_{i}^{\prime}$. (This completes a loop, corresponding to the meridian of a component of $K$.) The apices of the two triangles point in different directions, as in Figure 3. As with punctured torus bundles, these layers of boundary triangles are stacked together, forming fans that correspond to syllables of the word $\Omega$.
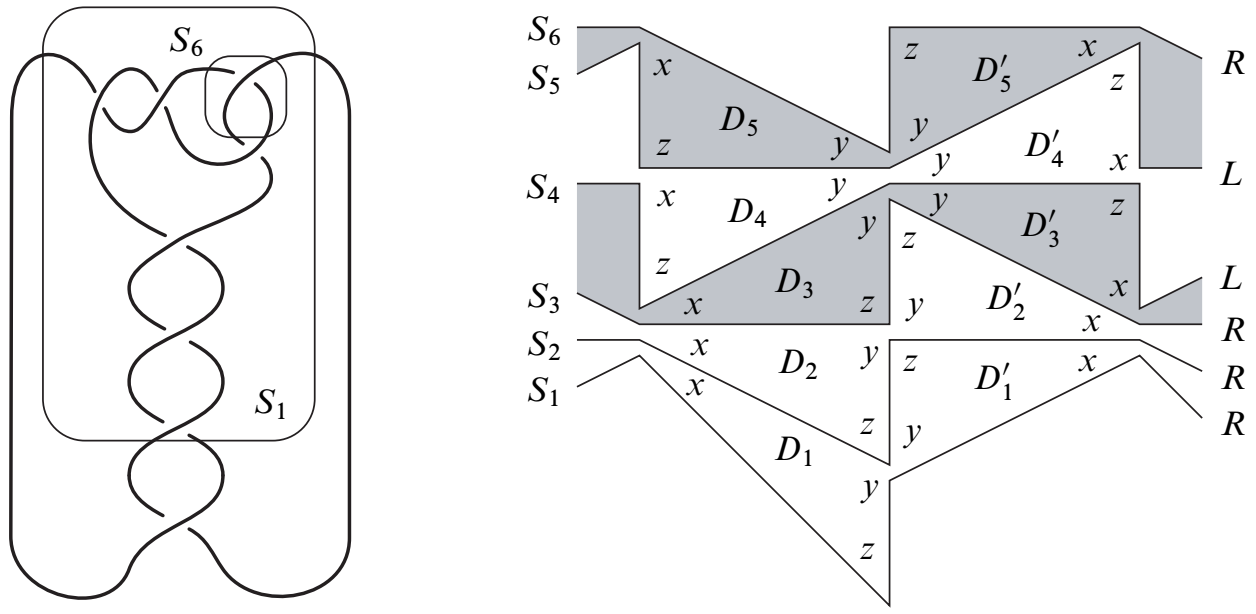

Figure 18: Left: the link $K(\Omega)$ corresponding to $\Omega=R^{3} L^{2} R$

Right: a cusp view of the product region between $S_{1}$ and $S_{c}$ 
The resulting cusp triangulation which corresponds to the product region of the link $K\left(R^{3} L^{2} R\right)$ is shown in Figure 18. As in Figure 4, the triangles are shown opened up, and the hinge layers are shaded. Observe that this picture is combinatorially equivalent to the corresponding picture for punctured torus bundles, quotiented by the hyperelliptic involution.

We have labeled the dihedral angles of the tetrahedra of $\Delta_{i}$ by numbers ("angles") $x_{i}, y_{i}, z_{i}$, following the same conventions as in Figure 4. Note that our choices of dihedral angles force the tetrahedra $D_{i}$ and $D_{i}^{\prime}$ to be isometric; this does not impede the goal of finding a geometric triangulation. In the sequel, we will not distinguish between $D_{i}$ and $D_{i}^{\prime}$.

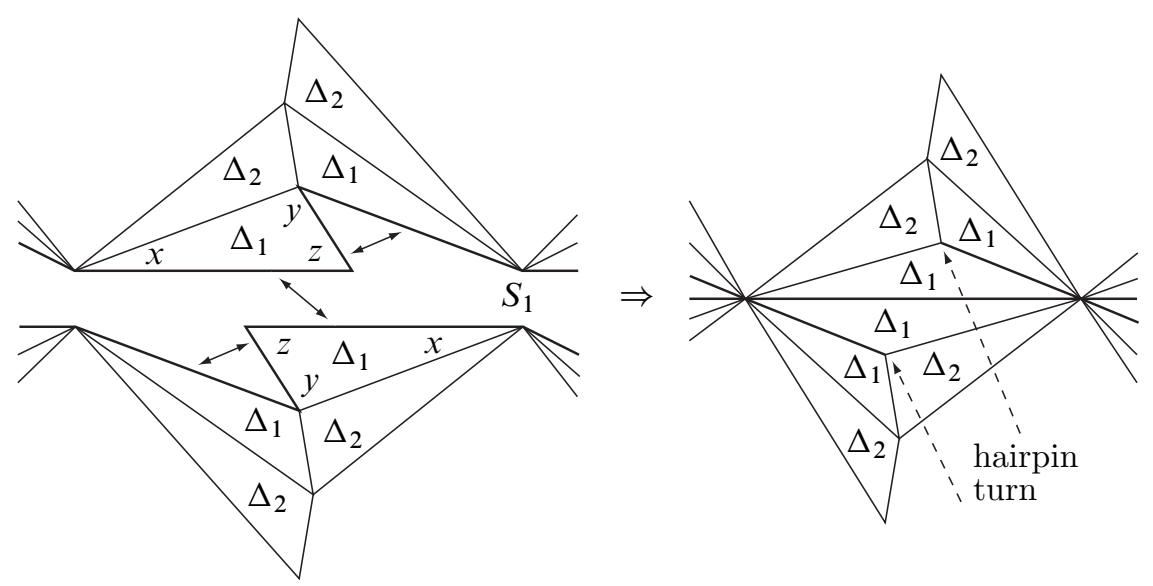

Figure 19: A cusp view of the folding that occurs at a clasp

When the pleated surface $S_{1}$ is folded to form a clasp, the zigzag line in which it intersects the cusp also becomes folded, creating a "hairpin turn." Because this folding procedure joins the punctures of $S_{1}$ in pairs, as in Figure 17, the boundary triangles on those punctures are also joined together. The resulting cusp triangulation in the neighborhood of $S_{1}$ can be seen in Figure 19. At the other end of the product region, the clasp of $S_{c}$ appears on the cusp in the same way.

Once we have folded the clasp surfaces as prescribed, the truncated vertices of the tetrahedra combine to form either a single torus that traverses the product region four times (in case $K$ is a knot), or two tori, each of which traverses the product region twice (in case $K$ is a two-component link). In either case, the local combinatorics are the same, and the affine equations that the dihedral angles of the tetrahedra must satisfy are derived in the same way. 
To find the hyperbolic structure on $\mathbb{S}^{3} \backslash K$, we will study the space $P$ of angle structures for the triangulation, ie the space of positive dihedral angles that line up correctly around each edge. We need to complete three steps:

(1) Parameterize $P$ and check that it is nonempty, as in Section 5.

(2) Show that a critical point of the volume functional on $P$ gives a complete hyperbolic metric, as in Section 6.

(3) Prove that at any point on the boundary of $\bar{P}$, the volume can be increased by unflattening the flat tetrahedra, as in Section 7-Section 9.

This will imply that the volume functional is maximized in the interior of $P$, guaranteeing a critical point and thus a hyperbolic metric. For each of the three steps, the argument is essentially the same as Guéritaud's.

\section{A.4 Angle structures and volume}

Following Section 5 of the main article, we will parameterize the dihedral angles of the tetrahedra by pleating angles on the pleated 4 -punctured spheres. Each sphere $S_{i}$ described above has a natural transverse orientation that points (equivalently) toward the inside of the link projection, toward increasing indices, and upward in Figure 18. Just as in Section 5, for any edge $e \subset S_{i}$, we can define the pleating angle $\alpha$ to be the (signed) external angle at $e$, with signs chosen so that $\alpha$ is positive whenever the angle above $e$ is less than $\pi$.

On the pleated sphere $S_{i+1}$ living between $\Delta_{i}$ and $\Delta_{i+1}$, this definition will give pleating angles

$$
-2 w_{i}, \quad 2 w_{i+1} \text { and } 2 w_{i}-2 w_{i+1},
$$

exactly as in (2). The clasp surface $S_{1}$, which borders $\Delta_{1}$ on one side and is folded on the other side, will have pleating angles $-\pi, 2 w_{1}$, and $\pi-2 w_{1}$, where the pleating angle of $-\pi$ corresponds to the hairpin turn in Figure 19. Thus, if we define $w_{0}=\pi / 2$ (even though there is no tetrahedron layer $\Delta_{0}$ ), the pleating angles on $S_{1}$ will be given by the same expressions as above. Similarly, setting $w_{c}=\pi / 2$ allows us to label the pleating angles on $S_{c}$ by the same expressions as in (2).

Lemma A.2 For $i=0, \ldots, c$, choose a parameter $w_{i}$, such that $w_{0}=w_{c}=\pi / 2$ and $w_{1}, \ldots, w_{c-1}$ satisfy the range, concavity, and hinge conditions (3). For each such choice of parameters, set the dihedral angles of the tetrahedra as in Table 1. Then

(1) for each $\Delta_{i}$, the angles $x_{i}, y_{i}, z_{i}$ are positive and add up to $\pi$,

(2) the dihedral angles around each edge add up to $2 \pi$, and 
(3) for each $S_{i}$, the pleating angles add up to 0.

Proof The range, concavity, and hinge conditions imply that all the tetrahedron angles $x_{i}, y_{i}, z_{i}$ are positive, and the claim that their sum is $\pi$ is immediate from Table 1 . For each pleated surface $S_{i}$, the pleating angles sum to 0 by construction. Therefore, it remains to check the angle sum around each edge $e$ of $\mathbb{S}^{3} \backslash K$.

If $e$ is not the core of $S_{1}$ or $S_{c}$, the combinatorial picture of Figure 18 is the same as the one for torus bundles. Thus, as in Section 5, each layer $\Delta_{i}$ contributes precisely the difference between the pleating angles of the neighboring surfaces, and the sum around $e$ simplifies to $2 \pi$. If $e$ is the core of a clasp, say the core of $S_{1}$, the left panel of Figure 19 shows that four sectors contribute dihedral angles at $e$ : two sectors that have angle $z_{1}$, plus two fans of angles above pleated surface $S_{1}$, each having dihedral angle $2 w_{1}$. Thus, because $z_{1}+2 w_{1}=\pi$, the total angle sum at $e$ is $2 \pi$.

By Lemma A.2, our triangulation will have an angle structure whenever we set $w_{0}=$ $w_{c}=\pi / 2$ and interpolate between these parameters in a way that satisfies the range, concavity, and hinge conditions. One can always do this graphically, by first fixing $w_{i}$ for the hinge indices and then connecting the hinges by pieces of parabolas, as in Figure 6 (this is where we use the hypothesis that $\Omega$ contains at least one hinge).

Lemma A.3 Let $P$ be the open affine polyhedron of angle structures for the triangulation of $\mathbb{S}^{3} \backslash K$, parameterized by sequences $\left(\frac{\pi}{2}, w_{1}, \ldots, w_{c-1}, \frac{\pi}{2}\right)$, as in Lemma A.2. Then a point of $P$ is a critical point of the volume functional $\mathcal{V}$ if and only if the corresponding tetrahedron shapes give a complete hyperbolic structure on $\mathbb{S}^{3} \backslash K$.

Just like Lemma 6.2, this is an instance of a much more general theorem of Rivin, Chan, and Hodgson [9; 22]. It is also possible to prove Lemma A.3 directly, using the same line of argument as in Lemma 6.2, although in the setting of two-bridge links this would require considering a number of special cases.

\section{A.5 Flat tetrahedra never maximize volume}

The proof that the maximum of $\mathcal{V}$ occurs in the interior of $P$ closely tracks Section $7-$ Section 9 of Guéritaud's paper. We begin by ruling out many types of degeneracies on $\partial \bar{P}$.

Lemma A.4 Let $\left(w_{1}, \ldots, w_{c-1}\right)$ be the point of $\bar{P}$ at which the volume functional $\mathcal{V}$ attains its maximum. Then $\left(w_{i}\right)$ has the following properties:

(1) For each $i$, if one of $x_{i}, y_{i}, z_{i}$ is 0 , then two are 0 , ie $\Delta_{i}$ is flat. 
(2) If $\Delta_{i}$ is flat, then $w_{i}=0$.

(3) If $\Delta_{i}$ is flat, then $i$ is a hinge index, not equal to 1 or $c-1$.

(4) If $\Delta_{i}$ is flat, then $i$ is adjacent to at least two consecutive identical letters.

(5) If both hinge layers at the ends of a syllable $R^{k}$ or $L^{k}$ are flat, then $k \geq 3$.

Proof All the discussion and results of Section 7 apply equally well in our context. Thus we have conclusion (1) as a restatement of Proposition 7.1. The domino effect of Proposition 7.2 also applies; in fact, it is clear from the proof of the Proposition that the domino effect works both forward and backward. Thus it does not matter that our word $\Omega$ is not cyclic.

Almost all the claims of (2)-(5) are proved in Section 7, either in Proposition 7.3 or in the discussion that follows. The one exception is the claim that $\Delta_{1}$ and $\Delta_{c-1}$ cannot flatten. This follows because, in the case of two-bridge links, $w_{0}=w_{c}=\pi / 2$. Thus setting $w_{1}$ or $w_{c-1}$ to 0 or $\pi / 2$ will trigger the domino effect and force all the tetrahedra to flatten, giving a volume of 0 .

It remains to prove that at any point $\left(w_{i}\right) \in \partial \bar{P}$ satisfying the properties of Lemma A.4, the volume will increase as we move into the interior of $P$. Following Guéritaud, we do this using the geometrical statement of Lemma 8.2. The proof of this lemma transfers perfectly to our context when the fan under consideration corresponds to a subword in the interior of $\Omega$. As it turns out, the same statement is even easier to prove when the degenerate layer is near the beginning or end of $\Omega$.

Lemma A.5 Recall the word $\Omega=R^{a_{1}} L^{a_{2}} \cdots$, and suppose that the hinge layer $\Delta_{a_{1}}$ has flattened, with $w_{a_{1}}=0$. Then the fan corresponding to $R^{a_{1}} L$ admits a complete Euclidean structure with boundary along $S_{a_{1}}$. Let $Q, P, T$ be the lengths of the segments of the broken line in which $S_{a_{1}}$ intersects the cusp, as in Figure 20. Then $Q<P+T$.

Of course, the analogous statement holds near the end of $\Omega$.

Proof Note that, by Lemma A.4, we have $a_{1} \geq 2$. For all $1 \leq i \leq a_{1}-2$, the parameter $w_{i}$ can vary in a small interval, and no generality is lost in assuming that $\partial \mathcal{V} / \partial w_{i}=0$. (Otherwise, volume is easy to increase.) Then, as in the proof of Lemma 8.2, the criticality of volume with respect to these parameters implies that the fan of $R^{a_{1}} L$ has a complete Euclidean structure. If $i>1$, the computation is the same as in Sublemma 6.4. If $i=1$, the angle $z_{0}$ of Figure 7 is replaced by the hairpin turn, and the factor $\frac{\sin y_{0}}{\sin x_{0}}$ disappears from the computation of $\exp \left(-\partial \mathcal{V} / \partial w_{1}\right)$. Thus we once again have 


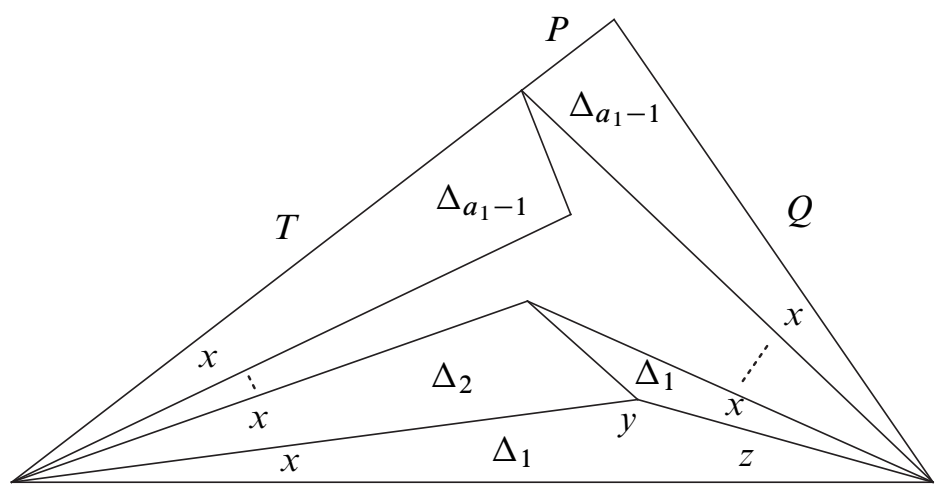

Figure 20: In a fan at the beginning of $\Omega, Q<P+T$.

$1=\exp \left(-\partial \mathcal{V} / \partial w_{1}\right)=|\psi(\alpha)|$, where $\psi$ is the reduced holonomy and $\alpha$ is a loop around the vertex at the hairpin turn.

In our situation, the fan of $R^{a_{1}} L$ is a (tessellated) Euclidean triangle, as in Figure 20, in which $Q$ and $P+T$ are two of the sidelengths. In the triangular fan, the angle opposite $Q$ is $x_{1}+\ldots+x_{a_{1}-1}$, while the angle opposite $P+T$ is $z_{1}+x_{1}+\ldots+x_{a_{1}-1}$. Thus, because its side is opposite the smaller angle, $Q<P+T$.

Armed with Lemma 8.2 and its analogue in Lemma A.5, we can complete the proof of Theorem A.1 by following the argument of Section 9. For each hinge index $j$ with $w_{j}=0$, we pick a vector along which to deform the neighboring parameters, in a way that will maximize the derivative of volume. Deforming in this optimal direction with speed $\varepsilon$, we can compute $\partial \mathcal{V} / \partial \varepsilon$ in terms of the geometry of the fan(s) that adjoin $\Delta_{j}$.

In most situations, the exponentiated derivative appears as a product of the exact same sine ratios as in Section 9. The only exception occurs when $j=2$ or $j=c-2$, because there are no tetrahedra corresponding to $w_{0}$ or $w_{c}$. If (without loss of generality) $j=2$, the angle $z_{0}$ is replaced by the hairpin turn, while the factor $\frac{\sin y_{0}}{\sin x_{0}}$ disappears from the computation of $P / T$, just as in the proof of Lemma A.5. Thus $\partial \mathcal{V} / \partial \varepsilon$ has the same expression in terms of $Q, Q^{\prime}, P, P^{\prime}, T, T^{\prime}$ as in Section 9.

In every case, the geometric statement $Q<P+T$, applied to two fans if necessary, implies that $\partial \mathcal{V} / \partial \varepsilon>0$. Therefore $\mathcal{V}$ is maximized at a critical point where all tetrahedron angles are positive, completing the proof of Theorem A.1. 


\section{Appendix B Applications}

Our construction of geometric triangulations using volume maximization methods has several corollaries that relate hyperbolic geometry (of bundles and links) to combinatorics (of the Farey complex and link diagrams).

\section{B.1 Volume estimates for bundles}

Theorem B.1 Let $V_{\varphi}$ be a once-punctured torus bundle defined by the cyclic word $\Omega=R^{a_{1}} L^{b_{1}} \ldots R^{a_{n}} L^{b_{n}}$. Then

$$
2 n v_{3} \leq \operatorname{Vol}\left(V_{\varphi}\right)<2 n v_{8}
$$

where $v_{3} \approx 1.0149$ is the volume of a regular ideal tetrahedron and $v_{8} \approx 3.6638$ is the volume of a regular ideal octahedron. Both of these bounds are sharp.

This is a sharp, quantitative version of Brock's result in [8], in the special case of punctured torus bundles. The upper bound is not new; it is a special case of [2, Corollary 2.4].

Proof To prove the lower bound on volume, we exhibit a particular angle structure. It follows from the proof of Theorem 1.1 that the volume of the complete hyperbolic structure on $V_{\varphi}$ is the global maximum of $\mathcal{V}$ over the closed polytope $\bar{P}$. Thus, for any $w \in \bar{P}, \mathcal{V}(w) \leq \operatorname{Vol}\left(V_{\varphi}\right)$.

We choose $w$ in the simplest possible manner: by letting $w_{i}=\frac{\pi}{3}$ for all $i$. It is easy to check that this choice of parameters satisfies at least the weak form of all the inequalities of (3). In other words, all angles are nonnegative, and $w \in \bar{P}$. Any nonhinge tetrahedron $\Delta_{i}$ will have one vanishing angle and will therefore have volume 0 . However, every hinge tetrahedron $\Delta_{i}$ will be a regular tetrahedron, with all angles $\frac{\pi}{3}$ and volume $v_{3}$. Thus $\mathcal{V}(w)=2 n v_{3}$.

When $\Omega=(R L)^{n}$, and thus all tetrahedra are hinges, this choice of angles will in fact give the complete structure on $V_{\varphi}$. These bundles, which are $n$-fold cyclic covers of the figure- 8 knot complement, have volume exactly $2 n v_{3}$. On the other hand, the bundles that contain some nonhinge tetrahedra have $\operatorname{Vol}\left(V_{\varphi}\right)>2 n v_{3}$, because $\mathcal{V}$ is maximized at a point where all tetrahedra have positive angles.

To prove the upper bound, we employ a Dehn surgery construction. For every Farey vertex $s$ which corresponds to a fan $R L^{*} R$ or $L R^{*} L$ (with $*>0$ ), drill a closed curve of slope $s$ out of the fiber at the corresponding level. (These are exactly the closed curves whose length was estimated in Section 10.) The resulting drilled bundle turns 
out to be an $n$-fold cyclic cover of the Borromean rings complement, with volume $2 n v_{8}$.

We can recover $V_{\varphi}$ by Dehn filling the extra cusps of the drilled bundle. Because volume goes down under Dehn filling, $\operatorname{Vol}\left(V_{\varphi}\right)<2 n v_{8}$. If we pick a word $\Omega$ with very long syllables, as in Section 10 , the resulting bundle $V_{\varphi}$ has volume arbitrarily close to the volume of this surgery parent.

Corollary B.2 Let $V_{\varphi}$ be a 4-punctured sphere bundle defined by the cyclic word $\Omega=R^{a_{1}} L^{b_{1}} \cdots R^{a_{n}} L^{b_{n}}$. Then

$$
4 n v_{3} \leq \operatorname{Vol}\left(V_{\varphi}\right)<4 n v_{8}
$$

\section{B.2 Volume estimates for links}

The volumes of link complements can also be estimated in terms of diagrams. We say that a link diagram $D$ is reduced if no single crossing separates $D$. Its twist number $\operatorname{tw}(D)$ is the number of equivalence classes of crossings, where two crossings are considered equivalent if there is a loop in the projection plane intersecting $D$ transversely precisely in the two crossings. When the diagram $D$ depicts a two-bridge link constructed from a braid, as in Figure 13, $\operatorname{tw}(D)$ is precisely the number of syllables of the word $\Omega$ describing the braid.

Theorem B.3 Let $D$ be a reduced alternating diagram of a hyperbolic two-bridge link $K$. Then

$$
2 v_{3} \operatorname{tw}(D)-2.7066<\operatorname{Vol}\left(\mathbb{S}^{3} \backslash K\right)<2 v_{8}(\operatorname{tw}(D)-1) .
$$

The upper bound is sharp, and the lower bound is asymptotically sharp.

There are known diagrammatic volume bounds for the general class of alternating links. On the lower side, Agol, Storm, and W Thurston proved that the volume of an alternating link is at least $\frac{v_{8}}{2}(\operatorname{tw}(D)-2)$ [3], improving an earlier bound due to Lackenby [14]. On the upper side, Agol and D Thurston proved an asymptotically sharp bound of $10 v_{3}(\operatorname{tw}(D)-1)$ [14, Appendix]. Numerically, Theorem B.3 improves the multiplicative constant in the lower bound from 1.8312 to 2.0299 and the multiplicative constant in the upper bound from 10.1494 to 7.3277 (in the special case of 2-bridge links).

Compared to its predecessors, Theorem B.3 is less general, but uses only very elementary methods. The proof in [3] relies in a fundamental way on Perelman's results about the monotonicity of volume under Ricci flow with surgery. By contrast, the lower bound in Theorem B.3 only relies on the explicit study of angled triangulations. 

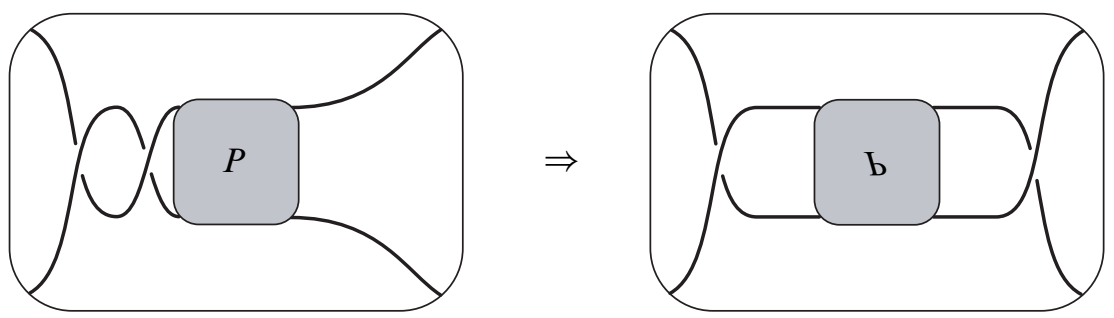

Figure 21: A flype in a diagram of a two-bridge link. The shaded rectangle $P$ is a pillowcase of the braid.

Proof First, we claim that it suffices to consider a diagram $D$ like the one in Figure 13, constructed from an alternating braid with one free strand. By the MenascoThistlethwaite flyping theorem [16], any pair of reduced alternating diagrams of $K$ are related by a sequence of flypes, as in Figure 21. It is easy to check that the twist number of a diagram is invariant under flypes. Thus the number of syllables of the word $\Omega$ is the twist number of any reduced alternating diagram of $K$.

Lower bound Suppose that the link is defined by the word $\Omega=R^{a_{1}} L^{a_{2}} \cdots R^{a_{n}}$ (the parity of $n=\operatorname{tw}(D)$ and the letter of the first and last syllables are unimportant). As in the proof of Theorem B.1, we explicitly choose a point $w=\left(\frac{\pi}{2}, w_{1}, \ldots, w_{c-1}, \frac{\pi}{2}\right)$ of $\bar{P}$. However, the concavity condition of (3) does not allow us to set $w_{i}=\frac{\pi}{3}$ when $i$ is too close to 0 or $c$. Instead, we proceed as follows. We let $w_{i}=\frac{\pi}{3}$ for all $a_{1} \leq i \leq c-a_{n}$. For the indices of the first and last fans, we interpolate linearly between $\frac{\pi}{2}$ and $\frac{\pi}{3}$. As before, it is easy to check that these values of the parameters make all tetrahedron angles nonnegative, and give us a point of $\bar{P}$.

When $i$ is a hinge and $a_{1}<i<c-a_{n}$, the two tetrahedra of $\Delta_{i}$ have all dihedral angles $\frac{\pi}{3}$, and volume $v_{3}$. For $n \geq 3$, there are exactly $n-3$ hinge indices of this type. When $i=a_{1}$ or $i=c-a_{n}$, we can compute from Table 1 that the three angles of $\Delta_{i}$ are

$$
\frac{\pi}{3}+t_{i}, \quad \frac{\pi}{3}-t_{i} \quad \text { and } \quad \frac{\pi}{3}, \quad \text { where } \quad t_{i}=\left|w_{i+1}-w_{i-1}\right| \leq \frac{\pi}{6} .
$$

By Proposition 6.6, the volume defined by these angles is smallest at the extreme value of $t_{i}=\frac{\pi}{6}$, when the three angles are $\frac{\pi}{2}, \frac{\pi}{3}, \frac{\pi}{6}$. Still assuming $n \geq 3$, by Formula (4) the four tetrahedra in the two terminal hinge layers each have volume at least $0.84578 \ldots$ Putting it all together gives

$$
\mathcal{V}(w)>2 v_{3}(\operatorname{tw}(D)-3)+4 \times 0.84578>2 v_{3} \operatorname{tw}(D)-2.7066 .
$$

(As a special case, if $n=2, \mathcal{V}(w)>2 \times 0.84578$ also satisfies the theorem.) 
To prove that this bound is asymptotically sharp, let $\Omega=(R L)^{k}$, for large $k$. Then $\operatorname{tw}(D)=2 k$, and the triangulation of $K(\Omega)$ consists of $2(\operatorname{tw}(D)-1)$ tetrahedra, all of them hinges. Since the volume of an ideal tetrahedron is bounded above by $v_{3}$, $\operatorname{Vol}\left(\mathbb{S}^{3}-K\right) \leq 2 v_{3}(\operatorname{tw}(D)-1)$, a value whose ratio to the lower bound of the theorem approaches 1 as $\operatorname{tw}(D)$ gets large.
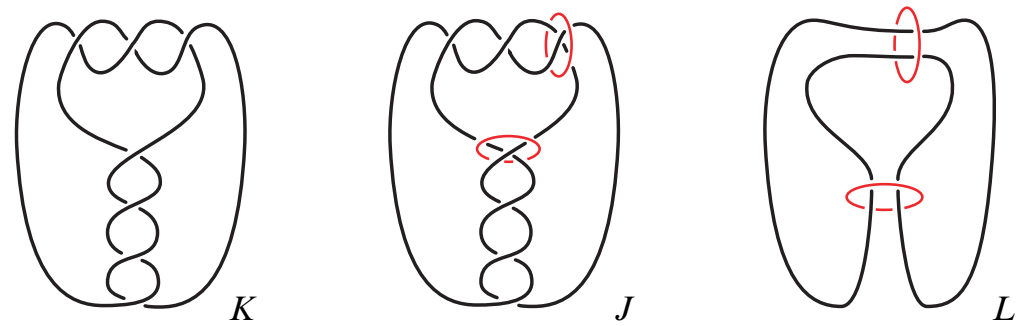

Figure 22: The construction of an augmented 2-bridge link $L$. When $\operatorname{tw}(D)=2, L$ is the Borromean rings.

Upper bound The proof of the upper bound uses the same surgery argument that Lackenby, Agol, and Thurston used for general alternating links [14], and the improved estimate comes from the special structure of 2-bridge links.

Let $D$ be a diagram as in Figure 13. Recall that each syllable of $\Omega$ corresponds to a twist region where two strands of the braid wrap around each other. For every twist region, we add an extra link component (called a crossing circle) encircling the two strands of $K$, obtaining a hyperbolic link $J$ [14]. (See Figure 22.) Every crossing circle of $J$ bounds a crossing disk that is punctured by the two strands of $K$. Because twice-punctured disks are totally geodesic [1], we can untwist all the crossings in the twist region and obtain a new link $L$, called an augmented link, whose volume is equal to that of $J$.

When $K$ is a two-bridge link, $L$ has the following alternate description. Start with $(\operatorname{tw}(D)-1)$ copies of the Borromean rings, cut each one along a crossing disk, and glue the copies together in a linear fashion. Volume is additive under this operation [1]. Thus $\operatorname{Vol}\left(\mathbb{S}^{3} \backslash L\right)=2 v_{8}(\operatorname{tw}(D)-1)$, since the Borromean rings have volume $2 v_{8}$. Since $K$ is obtained by Dehn filling the crossing circles of $J$, we have

$$
\operatorname{Vol}\left(\mathbb{S}^{3} \backslash K\right)<\operatorname{Vol}\left(\mathbb{S}^{3} \backslash J\right)=\operatorname{Vol}\left(\mathbb{S}^{3} \backslash L\right)=2 v_{8}(\operatorname{tw}(D)-1) .
$$

By choosing a link with many crossings in each twist region, one can get $\operatorname{Vol}\left(\mathbb{S}^{3} \backslash K\right)$ arbitrarily close to this upper bound. 


\section{B.3 Hyperbolic geodesics seen in the projection plane}

For any link diagram $D$, a crossing arc is a segment perpendicular to the projection plane that connects the upper strand of a crossing to the lower strand of the same crossing. Morwen Thistlethwaite has conjectured that in any reduced alternating diagram of a hyperbolic link $K$, every crossing arc is isotopic to a hyperbolic geodesic. As a consequence of Theorem A.1, we can prove this in the case of two-bridge links.

Theorem B.4 Let $D$ be a reduced alternating diagram of a hyperbolic two-bridge link $K$. Then every crossing arc of $D$ is isotopic to an edge in the Sakuma-Weeks triangulation of $\mathbb{S}^{3} \backslash K$, and thus to a geodesic.

In fact, still more is true: each edge of the triangulation is dual to a face of the Ford-Voronoi domain of $\mathbb{S}^{3} \backslash K[5]$.

Proof We begin by observing that the statement is true for a diagram $D_{0}$ as in Figure 13, constructed from an alternating braid with one free strand. Every crossing of $D_{0}$ corresponds to a 4-punctured sphere pleated along edges of our triangulation. As Figure 15 illustrates, the crossing arc of any crossing is isotopic to one of the edges.

As it turns out, the diagram $D_{0}$ is not overly special. Thistlethwaite has proved that every reduced alternating diagram $D$ of a two-bridge link is standard: that is, $D$ can also be constructed from an alternating 4-string braid, although not necessarily with a free strand [24, Theorem 4.1]. Furthermore, by the Menasco-Thistlethwaite flyping theorem [16], we can get from $D_{0}$ to $D$ by performing a sequence of flypes along the pillowcases of the braid. (See Figure 21.) During each flype, the diagram loses a crossing whose crossing arc is isotopic to an edge $e$ of the triangulation, and gains another crossing, whose arc is isotopic to an edge $e^{\prime}$ of the same slope as $e$. Thus the crossing arcs of every diagram are isotopic to geodesics.

\section{References}

[1] C C Adams, Thrice-punctured spheres in hyperbolic 3-manifolds, Trans. Amer. Math. Soc. 287 (1985) 645-656 MR768730

[2] I Agol, Small 3-manifolds of large genus, Geom. Dedicata 102 (2003) 53-64 MR2026837

[3] I Agol, P Storm, W Thurston, Lower bounds on volumes of hyperbolic Haken 3manifolds arXiv:math.DG/0506338

[4] H Akiyoshi, On the Ford domains of once-punctured torus groups, Sūrikaisekikenkyūsho Kōkyūroku (1999) 109-121 MR1744475 
[5] H Akiyoshi, M Sakuma, M Wada, Y Yamashita, Ford domains of punctured torus groups and two-bridge knot groups, from: "Knot Theory: dedicated to 70th birthday of Prof. Kunio Murasugi”, (M Sakuma, editor) (2000)

[6] H Akiyoshi, M Sakuma, M Wada, Y Yamashita, Jørgensen's picture of punctured torus groups and its refinement, from: "Kleinian groups and hyperbolic 3-manifolds (Warwick, 2001)”, London Math. Soc. Lecture Note Ser. 299, Cambridge Univ. Press, Cambridge (2003) 247-273 MR2044553

[7] P Alestalo, H Helling, On torus fibrations over the circle, Sonderforschungsbereich SFB-343 (1997)

[8] J F Brock, Weil-Petersson translation distance and volumes of mapping tori, Comm. Anal. Geom. 11 (2003) 987-999 MR2032506

[9] K Chan, Constructing hyperbolic 3-manifolds, Undergraduate thesis, University of Melbourne (2002) with C Hodgson

[10] F Guéritaud, Triangulated cores of punctured-torus groups arXiv: math.GT/0605481

[11] H Helling, The trace fields of a series of hyperbolic manifolds, Sonderforschungsbereich SFB-343 (1999)

[12] T Koch, Fordsche Fundamentalbereiche hyperbolischer einfach-punktierter TorusBündel, Sonderforschungsbereich SFB-343 (1999)

[13] M Lackenby, The canonical decomposition of once-punctured torus bundles, Comment. Math. Helv. 78 (2003) 363-384 MR1988201

[14] M Lackenby, The volume of hyperbolic alternating link complements, With an appendix by Ian Agol and Dylan Thurston, Proc. London Math. Soc. (3) 88 (2004) 204-224 MR2018964

[15] W Menasco, Closed incompressible surfaces in alternating knot and link complements, Topology 23 (1984) 37-44 MR721450

[16] W Menasco, M Thistlethwaite, The classification of alternating links, Ann. of Math. (2) 138 (1993) 113-171 MR1230928

[17] J Milnor, Hyperbolic geometry: the first 150 years, Bull. Amer. Math. Soc. (N.S.) 6 (1982) 9-24 MR634431

[18] Y N Minsky, The classification of punctured-torus groups, Ann. of Math. (2) 149 (1999) 559-626 MR1689341

[19] K Murasugi, Knot theory and its applications, Birkhäuser, Boston (1996) MR1391727

[20] W D Neumann, D Zagier, Volumes of hyperbolic three-manifolds, Topology 24 (1985) 307-332 MR815482 
[21] J-P Otal, Le théorème d'hyperbolisation pour les variétés fibrées de dimension 3, Astérisque (1996) $\mathrm{x}+159$ MR1402300

[22] I Rivin, Euclidean structures on simplicial surfaces and hyperbolic volume, Ann. of Math. (2) 139 (1994) 553-580 MR1283870

[23] M Sakuma, J Weeks, Examples of canonical decompositions of hyperbolic link complements, Japan. J. Math. (N.S.) 21 (1995) 393-439 MR1364387

[24] M B Thistlethwaite, On the algebraic part of an alternating link, Pacific J. Math. 151 (1991) 317-333 MR1132393

[25] Y Colin de Verdière, Un principe variationnel pour les empilements de cercles, Invent. Math. 104 (1991) 655-669 MR1106755

DMA, École normale supérieure, CNRS

45 rue d'Ulm, 75005 Paris, France

D Futer: Mathematics Department, Michigan State University East Lansing, MI 48824, USA

gueritau@dma.ens.fr, dfuter@math.msu.edu

Proposed: Walter Neumann

Seconded: Jean-Pierre Otal, Joan Birman
Received: 10 November 2005

Revised: 29 July 2006 